\title{
Quantum mechanics based force field for carbon (QMFF-Cx) validated to reproduce the mechanical and thermodynamics properties of graphite
}

\author{
Tod A. Pascal, ${ }^{1}$ Naoki Karasawa, ${ }^{2}$ and William A. Goddard III ${ }^{1, a)}$ \\ ${ }^{1}$ Materials and Process Simulation Center, California Institute of Technology, Pasadena, \\ California 91125, USA and Graduate School of EEWS, Korea Advanced Institute of Science and \\ Technology, Daejeon 305-701, Korea \\ ${ }^{2}$ Department of Photonics Science, Chitose Institute of Science and Technology, Hokkaido 0668655, Japan
}

(Received 5 April 2010; accepted 2 June 2010; published online 6 October 2010)

\begin{abstract}
As assemblies of graphene sheets, carbon nanotubes, and fullerenes become components of new nanotechnologies, it is important to be able to predict the structures and properties of these systems. A problem has been that the level of quantum mechanics practical for such systems (density functional theory at the PBE level) cannot describe the London dispersion forces responsible for interaction of the graphene planes (thus graphite falls apart into graphene sheets). To provide a basis for describing these London interactions, we derive the quantum mechanics based force field for carbon (QMFF-Cx) by fitting to results from density functional theory calculations at the M06-2X level, which demonstrates accuracies for a broad class of molecules at short and medium range intermolecular distances. We carried out calculations on the dehydrogenated coronene (C24) dimer, emphasizing two geometries: parallel-displaced $\mathrm{X}$ (close to the observed structure in graphite crystal) and PD-Y (the lowest energy transition state for sliding graphene sheets with respect to each other). A third, eclipsed geometry is calculated to be much higher in energy. The QMFF-Cx force field leads to accurate predictions of available experimental mechanical and thermodynamics data of graphite (lattice vibrations, elastic constants, Poisson ratios, lattice modes, phonon dispersion curves, specific heat, and thermal expansion). This validates the use of M06-2X as a practical method for development of new first principles based generations of QMFF force fields. (C) 2010 American Institute of Physics. [doi:10.1063/1.3456543]
\end{abstract}

\section{INTRODUCTION}

Empirical force fields ${ }^{1-8}(\mathrm{FFs})$ have been successful at describing many condensed phase materials and biological systems; however these FFs may not be sufficiently accurate for designing and predicting the properties of new materials. ${ }^{9}$ Thus it would be most useful to have FFs derived from quantum mechanics $(\mathrm{QM})$ since $\mathrm{QM}$ could be applied to structures and combinations of elements never before studied experimentally. Indeed QM can be trusted to provide accurate predictions of the valence forces and structures needed for such FFs. However, current generations of QM have difficulty in predicting accurately the attractive long range London dispersion $\left(1 / R^{6}\right)$ interactions. ${ }^{10}$ For example, the Perdew-Burke-Ernzerhof (PBE) flavor of density functional theory (DFT), the standard for QM on condensed phase structures, finds no minimum in the interactions between the graphene sheets in graphite, leading an infinite $c$ lattice parameter.

Recently, Zhao and Truhlar ${ }^{11}$ developed the new MOX (Refs. 11 and 12) generation of density functionals, and showed that the M06-2X functional gives excellent accuracy in describing weakly interacting, dispersion dominated systems (errors of $\sim 0.25 \mathrm{kcal} / \mathrm{mol}$ ). In this paper, we test the

\footnotetext{
a) Author to whom correspondence should be addressed. Electronic mail: wag@wag.caltech.edu.
}

utility of using M06-2X to develop QM based FFs, using as our test system, carbon. Since M06-2X has not yet been incorporated into periodic QM software for calculating graphite directly, we studied the dimer of dehydrogenated coronene (DHC) (C24) as a model system, considering the configurations most relevant to graphite. This leads to the QMFF-Cx FF, which we then use to calculate various mechanical, spectroscopic, and thermodynamic quantities of graphite crystal.

We focus on graphite partly due to the current interest in graphene ${ }^{13}$ and carbon nanoribbons ${ }^{14}$ as the foundation for future electronics. Since the required time and length scales for simulations on such devices are far too large for QM methods, it is essential to obtain accurate FF for predicting the structural and thermodynamic properties. Here, graphite is a natural starting point to derive the $\mathrm{C}-\mathrm{C}$ van der Waals (vdW) terms since the interactions between the planes can be considered as pure $\mathrm{vdW}$ in nature. In addition, experimental data of the $c$ lattice parameter are available down to $\sim 4 \mathrm{~K}$, providing valuable information on the equilibrium $\mathrm{C}-\mathrm{C}$ distance $\left(R_{e}\right)$. In addition, new experimental data have become available for the interaction energy between the planes providing unambiguous bond energy $\left(D_{e}\right)$ information.

In order to provide the most accurate validation of QMFF-Cx for graphite, it is important to compare to the experimental values at $0 \mathrm{~K}$. Section II outlines the selection 
of experimental properties and the extrapolation to $0 \mathrm{~K}$. Section III describes the process used to obtain the QMFF-Cx FF for graphite. Section IV discusses the predictions of the less well-characterized properties (e.g., C44 and C13). Section $\mathrm{V}$ reports a more detailed analysis of properties for graphite based on the predicted FF. This includes phonon dispersion curves and prediction of thermodynamic properties. Section VI considers the discrepancy between experimental values of $\mathrm{C} 44$ (which differ by a factor of 15) and the comparison with available theory.

\section{EXPERIMENTAL DATA FOR GRAPHITE}

\section{A. Crystal structure}

The experimental space group of graphite is $\mathrm{P}_{3} / \mathrm{mmc}$ $\left(D_{6 h}^{14}\right)$, which assumes that the graphene layers are flat. The lattice parameters at $25{ }^{\circ} \mathrm{C}$ are ${ }^{15} \quad \alpha=2.4612 \AA$ and $c$ $=6.7090 \AA$. To convert the lattice parameters at low temperature, we used the thermal expansion data from Ref. 16 $(20-270 \mathrm{~K})$, extrapolated and interpolated to obtain $\delta a=-0.0072 \AA$ and $\delta c=+0.0369 \AA$ for the total change from 0 to $298 \mathrm{~K}$. This leads to

$$
\begin{aligned}
& a(0 \mathrm{~K})=2.4684 \AA, \\
& c(0 \mathrm{~K})=6.6721 \AA .
\end{aligned}
$$

\section{B. Elastic constants}

A complete study of the elastic constants of compression annealed pyrolytic graphite (at room temperature) was carried out by Blakslee et al. ${ }^{17}$ The elastic constants $\mathrm{C} 11$ and $\mathrm{C} 12$ relate to in-plane deformations, $\mathrm{C} 13$ involves coupling of stress in the plane to spacing between the planes, C33 is a direct measure of the force constant for the graphenegraphene vdW interactions, and $\mathrm{C} 44$ relates to shear of graphene planes with respect to each other. The quantities $\mathrm{C} 11, \mathrm{C} 12, \mathrm{C} 33$, and $\mathrm{C} 44$ are derived from direct experiments; however, $\mathrm{C} 13$ is indirect.

The biggest variations in the elastic constants for graphite are found for $\mathrm{C} 44$ where values from 0.18 to $0.35 \mathrm{GPa}$ have been obtained from mechanical studies ${ }^{17}$ on compression annealed samples (the values were independent of external compression), while neutron scattering studies ${ }^{18,19}$ lead to values of $\mathrm{C} 44=4.2 \pm 0.2$ and $4.6 \pm 0.2 \mathrm{GPa}$. The neutronirradiated samples are referred to as "dislocation-free graphite" since it is believed that neutron damage (or impregnation with boron) impedes dislocation motion, leading to parameters appropriate for the intrinsic system. ${ }^{18,20,21}$ Boron implantation also leads to the larger values of $\mathrm{C} 44 .{ }^{20,21}$ From measurements of the specific heat at low temperatures, Komatsu $^{22}$ determined the $\mathrm{C} 44$ of natural graphite to be 4.05 and $4.52 \mathrm{GPa}$, while Bowman and Krumhans ${ }^{23}$ calculated a value of 2.3 by analyzing the specific heat at low temperatures.

There is evidence against this belief that low values for C44 from mechanical studies are due to dislocation migration. In studies down to $2 \mathrm{~K}$, Gauster and Fritz ${ }^{24}$ observed a nearly constant low value of $\mathrm{C} 44=0.18$, whereas if the low value was due to dislocations, one would have expected an increase for temperatures too low to activate the dislocations. Similarly, Ayasse et al. ${ }^{21}$ measured C44 down to $0.1 \mathrm{~K}$ and found no evidence of the hardening expected if the low C44 were due to dislocation motions.

In addition to the discrepancies in the $\mathrm{C} 44$, experimental values of $\mathrm{C} 13$ have large deviations $(39.5 \pm 40)$ and are obtained indirectly. Indeed, in the mechanical studies of Blakslee, ${ }^{17}$ it was not possible to measure $\mathrm{C} 13$ directly nor was it possible to measure the related compliance S13 in ultrasonic experiments. Instead, S13 was obtained by measuring the Poisson ratio in static experiments and $\mathrm{C} 13$ was calculated using

$$
C_{13}=-S_{13} /\left[S_{33}\left(S_{11}+S_{12}\right)-2 S_{13}^{2}\right]
$$

leading to $\mathrm{C} 13=15 \pm 5 \mathrm{GPa}$ at room temperature. In the temperature dependence studies of Gauster and Fritz, ${ }^{24} \mathrm{C} 13$ could be obtained only from crosscut samples $\left(45^{\circ}\right.$ from the $c$-axis), leading to a velocity of sound $v$ given by

$$
\begin{aligned}
p v^{2}= & \frac{1}{4}\left\{C_{11}+C_{33}+2 C_{44}\right. \\
& \left.-\sqrt{\left(C_{11}-C_{33}\right)^{2}+4\left(C_{13}+C_{44}\right)^{2}}\right\},
\end{aligned}
$$

where $p$ is the density. Since C11 is much larger than C13, this leads to considerable uncertainty in $\mathrm{C} 13$ (the uncertainty is comparable to the magnitude). Interestingly, the values for $\mathrm{C} 13$ deduced from these experiments are independent of temperature from 0 to $250 \mathrm{~K}$ and then change rapidly for higher temperature.

The temperature dependence of the elastic constants was measured by Gauster and Fritz ${ }^{24}$ on compression annealed graphite from 4 to $300 \mathrm{~K}$. We use these changes to better compare our $\mathrm{FF}$ results at $0 \mathrm{~K}$. As before, the values for $\delta \mathrm{C} 11, \delta \mathrm{C} 12, \delta \mathrm{C} 33$, and $\delta \mathrm{C} 44$ are obtained from independent experiments. However, $\delta \mathrm{C} 13$ is obtained from a complex elastic mode calculation, after subtracting the contributions from the other quantities (see Sec. IV C). As a result, $\delta \mathrm{C} 13$ has an uncertainty comparable to its magnitude. Our calculated elastic constants at $0 \mathrm{~K}$ is presented in Table S.1 of supplementary materials $(\mathrm{SM}){ }^{25}$

\section{Lattice vibrations}

With four atoms per unit cell, graphite has 12 vibrational branches. Raman and infrared (IR) studies lead to values of the $\Gamma$ point of the Brillouin zone $(k=0)$, as follows: ${ }^{26} E_{1 u}$ and $A_{2 u}$ are transverse acoustic (TA) and longitudinal acoustic (LA) (at $\left.0 \mathrm{~cm}^{-1}\right) ; E_{2 g}$ and $B_{2 g}$ are transverse (TO) and longitudinal (LO) modes for sheet-sheet interactions (at $\sim 10 \mathrm{~cm}^{-1}$ and $\left.\sim 140 \mathrm{~cm}^{-1}\right) ; A_{2 u}$ and $B_{2 g}$ are out-of-plane (ZO) crinklings of graphite (at $\sim 868 \mathrm{~cm}^{-1}$ ); $E_{1 u}$ and $E_{2 g}$ are in-plane ring modes (at $\sim 1588 \mathrm{~cm}^{-1}$ ).

The sheet-sheet modes $\left(E_{2 g}, B_{2 g}\right.$ or TO, LO) were also obtained from neutron scattering studies on "dislocationfree" graphite at room temperature, yielding ${ }^{18} 45$ and $126 \mathrm{~cm}^{-1}$ for the TO $\left(E_{2 g}\right)$ and $\mathrm{LO}\left(B_{2 g}\right)$ transition at the $\Gamma$ point (compared to $\sim 10$ and $140 \mathrm{~cm}^{-1}$ from IR and Raman). These neutron scattering studies lead to elastic constants of $\mathrm{C} 33=37.1 \pm 0.5 \mathrm{GPa}, \quad \mathrm{C} 44=4.6 \pm 0.2 \mathrm{GPa}, \quad$ and $\mathrm{C} 11$ $=1440 \pm 200 \mathrm{GPa}$. The value for $\mathrm{C} 33$ is consistent with the mechanical studies (36.5), but the other values are signifi- 
cantly higher than mechanical results $(\mathrm{C} 44=0.27$ and $\mathrm{C} 11$ $=1060$ ). We believe that this may be due to damage caused by neutron irradiation and have not used the neutron scattering values for the TO and $\mathrm{LO}$ modes in our fits.

\section{Thermal properties}

The specific heat of graphite has been studied extensively, with values obtained for both pure and impure graphite crystals (see the work of Nihira and Iwata ${ }^{27}$ and the references therein). The specific heat is reported as $1.92-2.06 \mathrm{cal} / \mathrm{mol} \mathrm{K}$ (Ref. 28) at $298 \mathrm{~K}$, and increases almost linearly with temperature up to $1500 \mathrm{~K}$, where it converges to $\sim 3.5 \mathrm{kcal} / \mathrm{mol} \mathrm{K}$. Below room temperature, the specific heat is sensitive to the purity of the graphite, the size of the crystal, and the differences in stacking faults. ${ }^{27}$ Above room temperature, it is believed that the specific heat is to be relatively insensitive to any of these effects.

Thermal expansion in graphite is highly anisotropic in graphite, with the observed lattice spacing in the $a b$ plane contracting with increasing temperature from 0 to $400 \mathrm{~K}$. This is presumably due to the increased fluctuations in the out-of-plane fluctuations by $-0.09 \mathrm{deg}^{-1} \mathrm{~K}$ at $30 \mathrm{~K}$ and -1.27 at $300 \mathrm{~K}$. On the other hand, the $c$ lattice increases rapidly in the $c$ direction by $+0.38 \mathrm{deg}^{-1} \mathrm{~K}$ at $30 \mathrm{~K}$ and 2.72 at room temperature due to the weak $\mathrm{vdW}$ interactions.

\section{COMPUTATIONAL DETAILS}

\section{A. QM calculations on the DHC dimer}

The M06-2X flavor of DFT is a new generation hybrid meta-generalized gradient approximation (GGA) exchangecorrelation functional with 54\% Hartree-Fock (HF) exchange that has been shown to describe weakly bound molecular complexes quite accurately $[0.44 \mathrm{kcal} / \mathrm{mol}$ mean absolute deviation (MAD) for dispersion dominated systems] near their equilibrium structures. ${ }^{11}$ Zhao and Truhlar ${ }^{29}$ used M06-2X to characterize the potential energy surface (PES) of coronene (C24H12) dimer. ${ }^{11}$ They found a minimum along the $x$ direction (denoted as PD-X for parallel displaced along the $x$-axis) that corresponds closely to the packing observed in graphite crystal and a second minimum (denoted as PD-Y) that for graphite would be a saddle point for shearing of the graphite planes between two PD-X minima. These calculations performed with the $6-31 \mathrm{G}+(\mathrm{d}, \mathrm{p})$ (double- $\zeta)$ basis set for geometry optimization and the $6-311+\mathrm{G}(2 \mathrm{df}, 2 \mathrm{p})$ (triple- $\zeta)$ basis for single point energies.

While coronene is frequently used as a model for graphite, the presence of the hydrogen induces additional electrostatic interactions due to charge polarization. Further, the development of a carbon FF using coronene is complicated by the need to simultaneously develop $\mathrm{H}-\mathrm{H}$ and $\mathrm{C}-\mathrm{H}$ parameters (or estimate them). One approach (used by Feller and Jordan $^{30}$ to estimate the strength of water-graphene interactions) is to repeat the calculations with flurocoronene (hydrogens replaced with flourines) and estimate the $\mathrm{C}-\mathrm{C}$ binding energy as the average of the two systems.

Our approach is to use DHC [C24, DHC: Fig. 1(a)] as the model. Here each edge carbon has an additional in-plane sigma bond with its neighbor (like 1-2 benzyne). This arm-

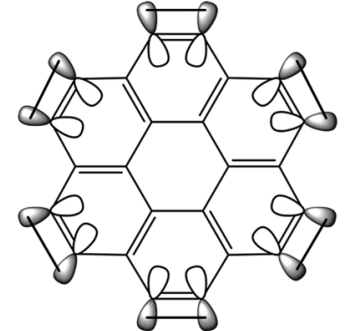

(a)

De-hydrogenated coronene (DHC) (b)

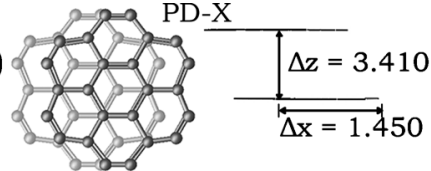

(c)

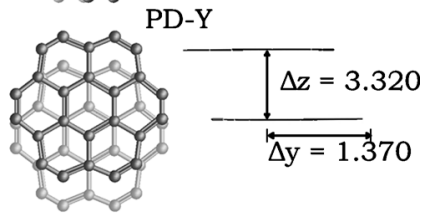

(d) $\frac{\square \Delta z=3.565}{\text { Eclipsed }}$
FIG. 1. (a) DHC unit used in this study. Since coronene has armchairlike edges, there are no dangling bonds: Each empty $\sigma$-orbital forms a bond with its neighbor. All carbon atoms are equivalent, making this molecule a reliable model for bulk graphite. (b) The optimized PD-X configuration from M06-2X/6-311G $+(2 d, 2 p)$ level of DFT. This is the most stable of the three configurations tested and is most similar to bulk graphite $(\Delta x$ $=1.425 \AA, \Delta z=3.33 \AA$ ). (c) The optimized PD-Y configuration, which is $0.66 \mathrm{kcal} / \mathrm{mol}$ higher than PD-X and represents a saddle point for sliding two DHC units. Our optimized geometry is similar to QM studies on the CD. (d) The high energy eclipsed structure is $6.05 \mathrm{kcal} / \mathrm{mol}$ higher than PD-X.

chair configuration inherent in coronene has no dangling bonds and every carbon has $s p 2$ type bonding.

Starting from the from the dimer coronene structures optimized by Zhao and Truhlar, we performed geometry optimization of DHC using the M06-2X DFT functional as implemented in the JAGUAR7.0 (Ref. 31) QM code. We chose the augmented polarized triple- $\zeta 6-311+\mathrm{G}(2 \mathrm{~d}, 2 \mathrm{p})$ basis sets of Pople and co-workers, ${ }^{32}$ a well balanced basis set providing a reasonable compromise between speed and accuracy (Fig. 2). Diffuse functions are known to be required for weakly bonded systems such as DHC dimer [Zhao and Truhlar $^{29}$ carried out extensive testing on the coronene dimer (CD)]. Hence, we tested the choice of basis set with single point calculations with eight other basis sets, with and without diffuse functions (Table S.III). In all calculations, we

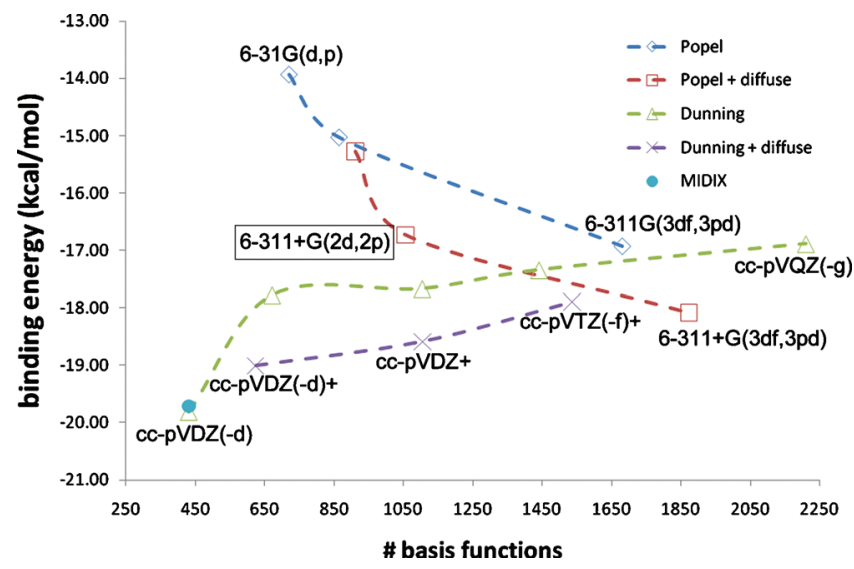

FIG. 2. Comparison of basis set for the counterpoise corrected binding energy of the PD-X configuration of DHC at the MO6-2X level DFT theory. Data are smooth using cubic splines (dashed lines) for presentation purposes. Single point energies in the various basis set were calculated using the minimum energy structure and the counterpoise corrections are then estimated. The $6-311+\mathrm{G}(2 \mathrm{~d}, 2 \mathrm{p})$ basis set was selected as a good compromise of speed (1056 basis functions) and accuracy. 

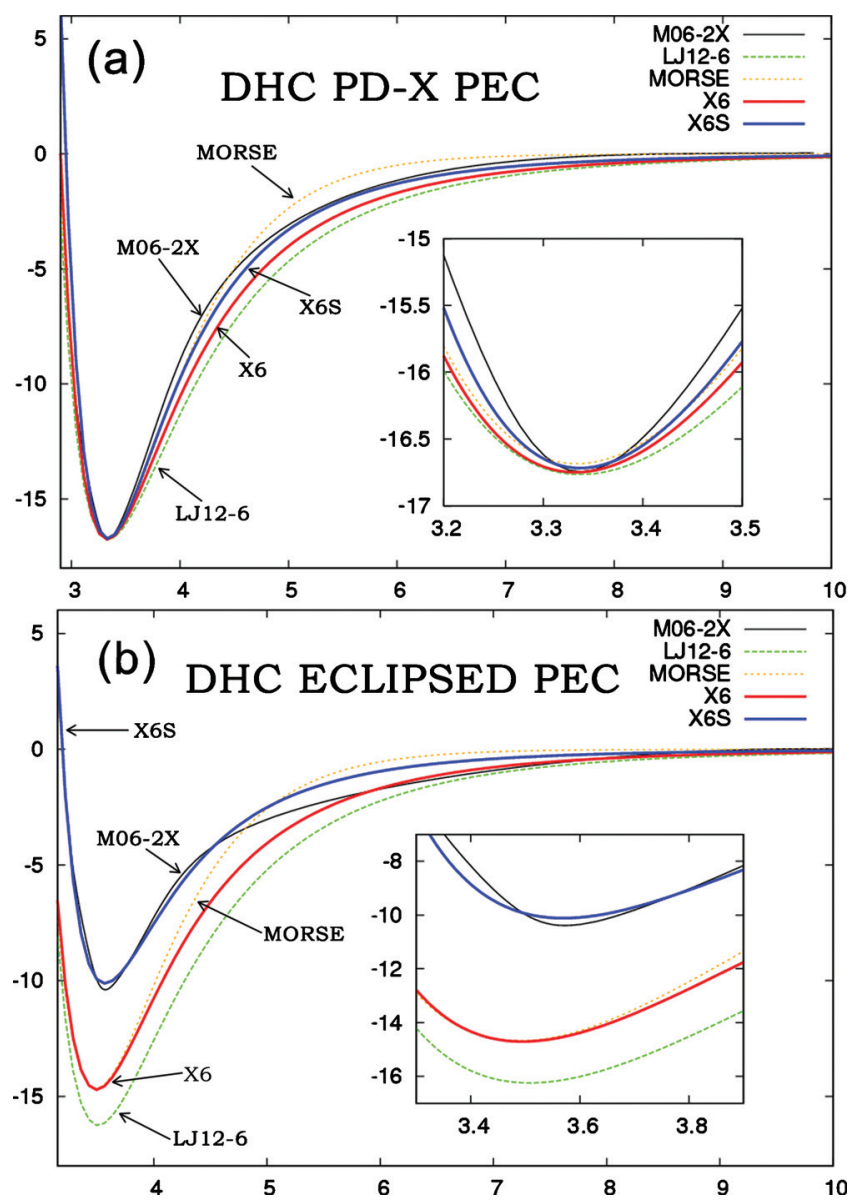

FIG. 3. Comparison of the QMFF-Cx and M06-2X vdW curves for the DHC (a) PD-X and (b) eclipsed geometries. The bottom of the PD-X curve was used in the fitting for all the QMFF-Cx potentials, while the PD-Y energy was used to fit the X6 and Morse potentials. The high energy, eclipsed geometry is well described only for the X6S (stretched X6) potential, which reproduces the $\mathrm{QM}$ value distance $(3.565 \AA)$ and energy $(-10.68$ $\mathrm{kcal} / \mathrm{mol})$. Inset: examination of equilibrium positions (3.4-3.8 $\AA$ ) for the both geometries.

account for bias due to basis set superposition error by using the counterpoise corrections method of Boys and Bernardi. ${ }^{33}$.

In-plane charge polarization was small but nonnegligible in the $\mathrm{CD}$ at the equilibrium geometries ${ }^{29}$ so we tested the charge polarization in DHC by computing atomcentered point charges using the electrostatic potential (ESP) charge model, the charge model 4 (CM4), ${ }^{34}$ and Mulliken populations $^{35}$ (Table S.II). Finally, potential energy curves (PECs) were calculated as a function of interplanar sheet separation $\Delta z$, with increments of $0.01 \AA$ (Fig. 3).

\section{B. The FF}

\section{1. vdW terms}

We determined the parameters of the nonbonded interactions in DHC using the following four potentials (Fig. 4):

(1) The Lennard-Jones 12-6 (denoted as LJ12-6),

$E_{\mathrm{vdw}}=D_{v}\left\{\rho^{-12}-2 \rho^{-6}\right\}$,

where $D_{v}$ is the well depth,

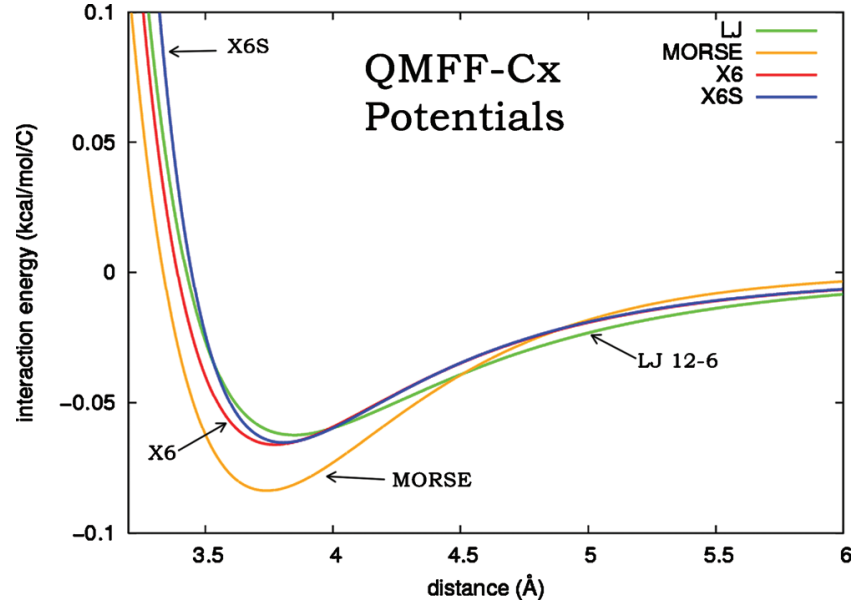

FIG. 4. PECs for the four potentials considered in this study. The parameters were all determined from the interaction of the DHC dimer from M06-2X DFT theory. The X6 and Morse potentials lead to predictions in excellent agreement with the QM for the PD-X and PD-Y geometrics, but are not able to reproduce the energetics of the high energy DHC eclipsed structure. The $\mathrm{X} 6 \mathrm{~S}$ potential has an additional parameter relating to the inner wall curvature parameter of 26.9 (compared to 20.7 for X6) and is able to reproduce the high energy structure.

$\rho=\frac{R_{v}}{R}$

is the scaled distance and $R_{v}$ is the well radius (distance between the carbons at the well minimum). Equation (1) leads to a force constant at the minimum of the form

$k_{v}=\left(\frac{\delta^{2} E}{\delta R^{2}}\right)_{R_{v}}=\frac{72 D_{v}}{R_{v}^{2}}$

so that the dimensionless force constant (i.e., curvature) is

$\bar{\kappa}=\frac{k_{v} R_{v}^{2}}{D_{v}}=72$.

(2) The Morse potential

$$
\begin{aligned}
E_{\mathrm{vdw}} & =D_{v}\left\{e^{-\left(\alpha_{v} / 2\right)(1-\rho)}-1\right\}^{2}-D_{v} \\
& =D_{v}\left\{e^{-\alpha_{v}(1-\rho)}-2 e^{-\left(\alpha_{v} / 2\right)(1-\rho)}\right\},
\end{aligned}
$$

where $\rho$ is given by Eq. (2) and $\alpha_{v}$ is the a parameter related to the force constant. This leads to a curvature $\bar{\kappa}=\alpha_{v} / 2$, which is the same as LJ12-6 if $\alpha_{v}=12$.

(3) Exponential-6 potential (denoted X6, also known as the Buckingham potential),

$E_{\mathrm{vdw}}=A e^{-C R}-b / R^{6}=\frac{D_{v}}{(\xi-6)}\left\{6 e^{\xi(1-\rho)}-\xi \rho^{-6}\right\}$,

where $\bar{\kappa}=6 \xi(\xi-7) /(\xi-6)$ is the curvature. The curvature of LJ12-6 is recovered if $\xi=13.772$.

(4) The stretched exponential potential (denoted as X6S), which has the same long range behavior as the original $\mathrm{X} 6$ in Eq. (1), 
TABLE I. Comparison of interaction energies $(\mathrm{kcal} / \mathrm{mol})$ for PD-X, PD-Y, and eclipsed DHC dimer structures between QM (counterpoise corrected $\left[\Delta E_{C p}\right]$ and uncorrected $[\Delta E]$ ) and QMFF-Cx. These can be compared to similar numbers on the CD from DFT and MP2 calculations. PD-X is topologically similar to the graphite structure, while PD-Y is a saddle point corresponding to the barrier between two adjacent PD-X minima. The interplane $z$ displacements for the QM structures are quoted in brackets.

\begin{tabular}{|c|c|c|c|c|c|c|c|c|c|c|}
\hline & \multicolumn{4}{|c|}{ QMFF-Cx ${ }^{\mathrm{a}}$ (DHC) } & \multicolumn{2}{|c|}{ M06-2X $\mathrm{X}^{\mathrm{b}}(\mathrm{DHC})$} & \multicolumn{2}{|c|}{ M06-2X $(C D)$} & \multicolumn{2}{|c|}{$\mathrm{MP}^{\mathrm{d}}(\mathrm{CD})$} \\
\hline & LJ12-6 [Eq. (1)] & Morse [Eq. (5)] & X6 [Eq. (6)] & X6S [Eq. (15)] & $\Delta E_{C p}$ & $\Delta E$ & $\Delta E_{C p}$ & $\Delta E$ & $\Delta E_{C p}$ & $\Delta E$ \\
\hline PD-X & -16.70 & -16.69 & -16.72 & -16.62 & -16.73 (3.410 ̊) & -22.10 & $-18.55(3.32 \AA)$ & -21.73 & -18.68 & -31.32 \\
\hline PD-Y & -16.65 & -16.04 & -16.00 & -16.10 & $-16.07(3.320 \AA)$ & -21.29 & $-17.88(3.33 \AA)$ & -20.98 & & \\
\hline Eclipsed & -16.24 & -15.62 & -15.56 & -10.51 & $-10.68(3.565 \AA)$ & -15.06 & $-9.77(3.66 \AA)$ & -12.64 & & \\
\hline${ }^{\mathrm{e}}$ Error & 5.59 & 4.94 & 4.88 & 0.20 & & & & & & \\
\hline
\end{tabular}

${ }^{\mathrm{a}}$ Parameters fit to QM with counterpoise corrections, vdW energies at the minimum of the PD-X and PD-Y curves, except for the stretched Exponential-6 (X6S), where the bottom of the eclipsed curve was also used.

${ }^{\mathrm{b}}$ Current work. 6-311+G(2d,2p) basis set.

${ }^{c}$ Calculations performed for the $\mathrm{CD}$ (Ref. 29). 6-31+G(d,p) for geometry optimization and 6-311+G(2df,2p) for single point calculations at minimum.

${ }^{\mathrm{d}}$ Calculations performed for the CD (Ref. 53). 6-31G* basis set.

${ }^{\mathrm{e}} \mathrm{L} 2$ norm of energy relative to M06-2X results, each structure weighted equally.

$E_{\text {disp }}=b / R^{6}=\frac{D_{v} \xi}{(\xi-6)} \rho^{-6}$

but a scaled short range Pauli repulsion,

$E_{\mathrm{rep}}=A e^{-(C \times D) R}=\frac{D_{v}}{(\xi-6)}\left\{\frac{6}{R} e^{\chi \xi \xi(1-\rho)}\right\}$,

where $\xi$ is the inner walls scaling factor $(\xi \geq 1)$, giving a dimensionless force constant

$\bar{\kappa}=\frac{6 \xi(\chi \xi-7)}{\xi-6}$.

When $\xi=1$ this leads to the original X6 potential.

\section{Valence terms}

The valence interactions were described using

- a Morse potential $E_{b}=D_{b}\left[e^{-\left(\alpha_{b} / 2\right)\left(1-\rho_{b}\right)}-1\right]^{2}+D_{b}$ for the $\mathrm{C}-\mathrm{C}$ bond stretch, where $k_{b}=2 D_{b} \alpha_{b}^{2}$ is the force constant;

- a cosine angle bend for the $\mathrm{C}-\mathrm{C}-\mathrm{C}$ interaction with angle-stretch $\left(k_{r \theta}\right)$ and stretch-stretch $\left(k_{r r}\right)$ coupling

$$
\begin{aligned}
E_{a}= & \frac{1}{2} C\left[\cos \theta-\cos \theta_{a}\right]^{2}+k_{r r}\left(R_{1}-R_{b}\right)\left(R_{2}-R_{b}\right) \\
& +D\left(\cos \theta-\cos \theta_{a}\right)\left[\left(R_{1}-R_{b}\right)+\left(R_{2}-R_{b}\right)\right],
\end{aligned}
$$

where $\vartheta_{a}$ is the equilibrium angle, $k_{\vartheta \vartheta}=C \sin ^{2} \vartheta_{a}$ is the diagonal angle force constant, and $k_{r \vartheta}=-D \sin \vartheta_{a}$ is the angle-stretch force constant;

- a twofold torsion

$E_{t}=\frac{1}{2} V_{t}(1-\cos 2 \theta)$,

where $\Phi$ is the torsional angle, $V_{t}$ is the barrier, and the minimum is for theta $\Phi=0$ (planar).

\section{Fitting procedure}

Table I: To optimize the nonbond parameters, we em- ployed least-squares fitting in a Newton-Raphson minimization scheme. Here, we let $\zeta$ be the set of observables we require the $\mathrm{FF}$ to reproduce, i.e.,

$$
\{\zeta\}=\left\{R, E, \frac{\partial E_{i}}{\partial R_{i}}\right\}
$$

is a function of the DHC plane-plane distance $(R)$ at equilibrium, the binding energy from $\mathrm{QM}(E)$, and the atomic forces $\left(\partial E_{i} / \partial R_{i}\right)$ for atom $i$. The per-atom forces were calculated from the QM wave function by numerical differentiation of the energy of $24(6 N$ where $N=4)$ perturbed geometries, moving each atom $\pm 0.05 \mathrm{bohr}$ in each $x / y / z$ direction.

We then minimize the residual function $R(\{\zeta\})$,

$$
R(\{\zeta\})=\sum_{i=1}^{N} w_{i}\left[R_{i}^{\mathrm{calc}}(\{\zeta\})-R_{i}^{\mathrm{obs}}(\{\zeta\})\right]^{2},
$$

where $N$ is the number of confirmations used in the fit and $w_{i}$ is the weighting factor. Typical values for weighting factor are 10 for the distances, 100 for the energies, and 250 for the forces.

The vdW interactions within a sheet lead to a tension that is balanced by the bond stretch forces. Hence we fixed the vdW terms and optimized the valence terms separately by direct minimization of the graphite crystal structure using the biased-Hessian method. ${ }^{36}$ We required zero stress within the plane while matching the $868 \mathrm{~cm}^{-1}$ (out-of-plane crinkling) and $1588 \mathrm{~cm}^{-1}$ (in-plane distortion) lattice modes. This allowed optimization of the $R_{b}, k_{b}, k_{\theta \theta}, k_{r \theta}$, and valence parameters. Due to redundancy, we did not optimize $D_{b}, k_{r r}$, or $\theta_{a}$.

The dispersive part of the $\mathrm{vdW}$ interactions for the $\mathrm{LJ}$ and X6 potentials were obtained using Ewald summations, with the accuracy bounded convergence criterion for the Ewald parameters. ${ }^{37}$ The convergence tolerance was $0.001 \mathrm{kcal} / \mathrm{mol}$, leading to a real space cutoff of $5.2 \AA$ and a reciprocal space cutoff of $k_{\text {cut }}$ of $1.4 \AA^{-1}$. For the Morse potential we used the fifth order taper function ensuring that the energy, and first and second derivatives are continuous as the potential goes smoothly to zero from 9.0 to $10.0 \AA$.

We used the 868 and $1588 \mathrm{~cm}^{-1}$ experimental lattice modes in our optimization protocol since the M06-2X func- 
tional is not yet implemented in periodic codes. We expect however that M06-2X would lead to similar values for these two lattice modes since other DFT functionals [for example, local density approximation (LDA) and GGA (Ref. 38)] give results in good agreement with experiment.

\section{Thermodynamic properties}

To calculate thermodynamic properties for a crystal requires summation over all phonon states of the system. Here we use a uniform grid with $N_{g}$ points along the $x, y$, and $z$ directions in reciprocal space (a total of $N_{g}{ }^{3}$ points) with standard harmonic quantum statistical parameters to calculate the total partition function from which the thermodynamic properties are calculated. These calculations used 1/24

$$
f(v)=\left\{\begin{array}{cc}
\frac{4 V}{c^{\prime}}\left(\frac{1}{v_{1}^{2}}+\frac{1}{v_{t}^{2}}\right) v \sin ^{-1}\left(\frac{v}{v_{g}}\right) & \text { for } v \leq v_{g}, \\
\frac{4 V}{c^{\prime}}\left(\frac{1}{v_{1}^{2}}+\frac{1}{v_{t}^{2}}\right) v \frac{\pi}{2} & \text { for } v \leq v_{g},
\end{array}\right.
$$

and

$$
f(v)= \begin{cases}\frac{V}{\pi c^{\prime} \kappa} x \int_{0}^{\sin ^{-1}\left(1 / \sqrt{1+\left(x_{0}(x)\right]}\right)} \frac{d \varphi}{\sqrt{1-x^{2}\left(1+\frac{x_{0}^{2}}{x^{2}} \sin ^{2} \varphi\right)}} & \text { for } v \leq v_{z}^{\prime}=\frac{\mu}{\pi}, \\ \frac{V}{\pi c^{\prime} \kappa}\left[1+\frac{x_{0}^{2}}{x^{2}}\right]^{-0.5} \int_{0}^{\pi / 2} \frac{d \varphi}{\sqrt{1-x^{2}\left(1+\frac{x_{0}^{2}}{x^{2}} \sin ^{2} \varphi\right)}} & \text { for } v \geq v_{z},\end{cases}
$$

of the Brillouin zone with proper weights to obtain the sum over the full Brillouin zone. The three zero frequency modes for $k=0$ are ignored in all calculations.

More accurate thermodynamic properties at lower temperature $(T<10 \mathrm{~K})$ can be obtained using the frequency distribution functions derived by Komatsu. ${ }^{45}$ These functions were derived from the equations for vibrations of thin plates with coupling terms between the plates. This approximation describes only the acoustic modes and is accurate for frequencies below about $130 \mathrm{~cm}^{-1}$. Thus for low temperatures (where contributions from high frequencies are small) it is adequate to use these functions for the thermodynamic properties. The frequency distribution functions for waves with polarization vectors in the plate are ${ }^{45}$ for waves with polarization vector out of the plate, where $x$ $=v / v_{z}^{\prime}$ and $x_{0}=\zeta / 4 \pi \kappa v_{z}^{\prime}$. QMFF-Cx X6 leads to the following parameters in these calculations: $c^{\prime}=c / 2=3.3325$ $\times 10^{-8} \mathrm{~cm}, V$ is the molar volume, $v_{1}$ is the longitudinal wave velocity in the plate, $v_{1}=\sqrt{C_{11} / \rho}=2.229 \times 10^{6} \mathrm{~cm} / \mathrm{s}$, $V_{t}$ is the transverse wave velocity in the plate, $V_{t}=\sqrt{C_{66} / \rho}$ $=1.433 \times 10^{6} \mathrm{~cm} / \mathrm{s}, \kappa$ is the bending modulus of the plate $=5.616 \times 10^{-3} \mathrm{~cm}^{2} / \mathrm{s}, \mu=\left(1 / c^{\prime}\right) \sqrt{C_{33} / \rho}=1.2704 \times 10^{13} \mathrm{~s}^{-1}$, and $\zeta=C_{44} / \rho=1.1562 \times 10^{9} \mathrm{~cm}^{2} / \mathrm{s}^{2}$.

Here, the bending modulus $\kappa$ was obtained by fitting the equation $v=\kappa k_{x}^{2} / 2 \pi$ to the frequencies calculated for the outof-the-plane modes at $k_{x}=0.1 k_{a}$. In this calculation, we used a single graphene sheet.

We also compare QMFF-Cx to a variety of experimental heat capacity data: for low temperatures $(<300 \mathrm{~K})$ we use the data of Desorbo and Tyler ${ }^{47}$ on pile graphite, Canadian natural graphite, and graphitized lampblack; from 300 to $500 \mathrm{~K}$, we used the data from JANAF (Ref. 48); from 750 to $2500 \mathrm{~K}$ we used the Hultgren ${ }^{46}$ data. To convert the calculated values of $C_{v}$ to $C_{p}$, we used the thermal expansion detailed in the next section.

\section{E. Thermal expansion}

In the harmonic approximation used above, the phonon frequencies do not depend on temperature; the graphite lattice parameters would therefore not depend on temperature. ${ }^{38}$ It is well established that as temperature increases, the motion of atoms in a crystal becomes increasingly anharmonic due to phonon-phonon coupling, usually leading to cell expansion. Hence the simple harmonic approximation breaks down at temperature above $10 \mathrm{~K}$. Strictly speaking, it is only valid at very low temperatures where atomic vibrations are mostly harmonic (the vibrations are purely harmonic in the limit of absolute zero).

These anharmonic effects can be included by means of the quasiharmonic approximation, which assumes that the internal degrees of freedom can be treated harmonically and the cell parameters optimized based on the free energy instead of the internal energy; the free energy captures the dependence of the phonon frequencies on the temperature. The quasiharmonic approximation has been applied successfully to the study of a variety of bulk systems ${ }^{49}$ and is valid up to temperatures as high as half the melting point of the material. 
We use the zero static internal stress approximation of Allan and co-workers ${ }^{50}$ as implemented in the GULP code ${ }^{51}$ for the free energy minimization, with a uniform grid $N_{g}$ $=20$ grid points (in each $x, y, z$ direction) in reciprocal space. The linear coefficient of thermal expansion $(\alpha)$ is then simply calculated from the numerical derivative of the two lattice parameters with temperature

$$
\begin{aligned}
& \alpha_{a}(T)=\frac{1}{a_{0}}\left(\frac{\partial a}{\partial T}\right)_{p}, \\
& \alpha_{c}(T)=\frac{1}{c_{0}}\left(\frac{\partial c}{\partial T}\right)_{p},
\end{aligned}
$$

where $a_{0}$ and $c_{0}$ are the lattice parameters at $0 \mathrm{~K}$. The calculated in-plane lattice constants are compared to available experimental data in Fig. 9.

\section{RESULTS}

\section{A. Structure and energies of DHC dimer}

The M06-2X binding energies for the PD-X, PD-Y and eclipsed DHC structures (Fig. 1) are shown in Table I. We find that the PD-X structure is the global minimum with an average binding energy (half of the total and counterpoise corrected binding energies) of $-19.41 \mathrm{kcal} / \mathrm{mol}$. This can be compared to calculations on the $\mathrm{CD}$ by (1) Zhao and Truhlar $^{29}$ using M06-2X, which gave $-20.1 \mathrm{kcal} / \mathrm{mol}$ and (2) DFT-D [BLYP-D (Ref. 54) and B97-D (Ref. 55)] studies, which employ empirical corrections for dispersion interactions, of -21.6 and $-21.5 \mathrm{kcal} / \mathrm{mol}$, respectively.

PD-Y is less stable than PD-X by $0.66 \mathrm{kcal} / \mathrm{mol}$, while the eclipsed structure is $6.05 \mathrm{kcal} / \mathrm{mol}$ less. These results are also similar to the $\mathrm{CD}$ calculations of Zhao and Truhlar who obtained $0.67 \mathrm{kcal} / \mathrm{mol}$ for PD-X/PD-Y and 8.78 for PD-X/ eclipsed. We note however that the average counterpoise correction for the three minima in DHC is $5.01 \mathrm{kcal} / \mathrm{mol}$, which is larger than found for coronene by Zhao and Truhlar $(3.02 \mathrm{kcal} / \mathrm{mol})$. A much larger correction of $12.64 \mathrm{kcal} / \mathrm{mol}$ was obtained ${ }^{53,56}$ for MP2 calculations on the PD-X structure of $\mathrm{CD}$ using the smaller $6-31 \mathrm{G}^{*}$ basis set, but after counterpoise correction the total binding energies are similar. We therefore selected the counterpoised corrected energies as the true value.

Comparing basis sets (Table S.III), we find some differences between the polarized basis sets of Pople et al. ${ }^{32,57}$ and the split valence, correlation-consistent basis sets of Dunning et al. ${ }^{58}$ We will primarily focus on the basis sets with diffuse functions since they correct deficiencies arising from the relatively inflexible valence shell functions in weakly interacting, dispersion dominated systems. ${ }^{59}$

We find reasonable agreement in the total binding energy (without counterpoise corrections) between both classes of basis sets for the PD-X (22.1 versus $22.8 \mathrm{kcal} / \mathrm{mol})$, PD-Y (21.0 versus $22.5 \mathrm{kcal} / \mathrm{mol})$, and eclipsed (14.7 versus $15.4 \mathrm{kcal} / \mathrm{mol}$ ) structures, with average deviations of $\pm 1.8 \mathrm{kcal} / \mathrm{mol}$. Similar agreement is obtained when considering the basis sets without diffuse functions, although the binding energies are $1-2 \mathrm{kcal} / \mathrm{mol}$ different.
The counterpoise corrected energies exhibit larger differences, however, with the Pople basis sets having an average of $0.8 \mathrm{kcal} / \mathrm{mol}$ more correction than Dunning's, these are consistent with other studies. ${ }^{59}$ As a result, the counterpoise corrected binding energies from the Pople set are $\sim 1.8 \mathrm{kcal} / \mathrm{mol}$ more stable than Dunning's. The much smaller MIDIX basis set of Truhlar and co-workers ${ }^{60}$ (432 basis functions for PD-X, compared to 1536 for Dunning augmented triple- $\zeta$ ) lead to uncorrected total binding energies larger by $8-10 \mathrm{kcal} / \mathrm{mol}$, but after counterpoise corrections lead to net binding energies similar to the other basis sets. This supports the counterpoise corrected energies as the most reliable.

Extrapolating the binding energies of the Dunning basis sets with a fourth order polynomial to the complete basis set limit yields total binding energies of $-16.3,-15.7$, and $-10.2 \mathrm{kcal} / \mathrm{mol}$ for PD-X, PD-Y, and the eclipsed structures, respectively. These energies are similar to the Pople 6-311 $+\mathrm{G}(2 \mathrm{~d}, 2 \mathrm{p})$ triple- $\zeta$ basis set $(-16.7,-16.1$, and -10.7 , respectively). Consequently, we select triple- $\zeta$ basis for FF fitting, as it leads to a good combination of accuracy and speed (1056 basis function for DHC dimer).

The largest Pople basis set use in this study is the $6-311+\mathrm{G}(3 \mathrm{df}, 3 \mathrm{pd})$ (1872 basis functions for the dimer) and can be considered a quadruple- $\zeta$ basis set. The energies obtained $(-18.1,-16.5$, and -12.0 , respectively) are much larger than the other basis sets and are not consistent with extrapolating from the double- $\zeta(6-31+\mathrm{G}(\mathrm{d}, \mathrm{p}))$ and triple- $\zeta$ (Fig. 2). This may indicate an imbalance in this basis set.

The atom-centered charges on the DHC monomer and equilibrium PD-X structure were evaluated using the ESP and Mulliken charge methods, and compared to the CM4 results on coronene reported by Zhao and Truhlar (Table S.II). Here we find two to ten times reduction in charge polarization in DHC monomer compared to coronene. Absolute charge transfer in the DHC dimer is also negligible $\left(<10^{-4} e^{-}\right.$ overall) and net 0 by symmetry. For internal consistency, we chose to include the ESP monomer charges during the FF fitting. These ESP charges were $-0.0025 e^{-}$for the inner 6 carbons, 0.012 the next 6 carbons, and -0.00475 the terminal 12 carbons. This Friedel-like oscillating charge ${ }^{61}$ is related to the wavelength of the highest occupied orbitals.

We calculate the interplanar distance to be $3.410 \AA$ for PD-X, $3.20 \AA$ for PD-Y, and $3.565 \AA$ for the eclipsed structure. The 3.3-3.6 $\AA$ interplanar distances in DHC is consistent with the range observed experimentally for stacked aromatic units ${ }^{62}$ and $3.336 \AA$ experimental value for graphite structure. The PD-X interplanar distance is consistent with values on the CD from the MP2/6-31G(d) studies of Ruuska and Pakkanen ${ }^{53}$ (3.41 $⿱$ ) and the BLYP-D/TZV-(2d,2p) studies of Grimme ${ }^{55}(3.40 \AA)$, although slightly larger than the Zhao-Truhlar value of $3.32 \AA$. Similarly the $1.45 \AA$ $\times$ displacement displacement in for PD-X DHC is closer to the $1.425 \AA$ in graphite than the $1.76 \AA$ reported by Zhao and Truhlar for coronene, indicating that DHC is indeed a better model for graphite than coronene. 


\section{B. QMFF-Cx parameters}

Table I shows that both X6 $(-16.72$ and $-16.00 \mathrm{kcal} /$ mol) and Morse potentials ( -16.69 and $-16.04 \mathrm{kcal} / \mathrm{mol})$ lead to excellent descriptions of the PD-X and PD-Y minima (QMs are -16.73 and -16.07 , respectively). By comparison, the Lennard-Jones (LJ) potential is too soft, leading to a PD-X/PD-Y splitting of only $0.05 \mathrm{kcal} / \mathrm{mol}$. The third parameter of the X6 $(\zeta=16.1)$ and Morse potentials $(\alpha=12.8)$ lead to the increased curvature required to distinguish these structure. Here, the dimensionless force constants $\bar{\kappa}$ are 87.0 for X6 and 82.2 for Morse, compared to 72 for LJ12-6.

All three standard functional forms deviate significantly from the M06-2X description as the graphene-graphene separations are increased, as shown in Fig. 3 (the PD-Y PEC is similar to PD-X). In particular, the M06-2X curve leads to $\sim 1 / R^{8}$ behaviors rather than the $1 / R^{6}$ behavior for the LJ12-6 and X6 QMFF-Cx potentials (the Morse potential exhibits an exponential behavior $>6 \AA$ from equilibrium). We believe that the $1 / R^{8}$ behavior in M06-2X is due to the data used to fit: The parameters of the M06 class of functionals contain only equilibrium distances and energies (the only experimental data available for the fit) with no information about the long range form of the potentials. Indeed, there is evidence that the M06-2X class of potentials has incorrect behavior far from equilibrium, ${ }^{29}$ prompting us to only fit the M06-2X data at the minima. Thus we consider that our FFs (Table II) are more accurate than M06-2X for larger separations. Here, experimental vibrational data for DHC or CD might be useful in establishing higher quality curvatures at the vdW minima.

\section{Comparison to experiment}

The $c$ lattice constant is directly related to the $\mathrm{vdW}$ radius and there is a good agreement with the experimental value at $0 \mathrm{~K}$ of 6.6721 for LJ12-6 (-0.25\%), Morse

TABLE II. Optimized FF parameters for graphite at $0 \mathrm{~K}$.

\begin{tabular}{|c|c|c|c|c|}
\hline Parameters & LJ12-6 & Morse & X6 & X6S \\
\hline \multicolumn{5}{|c|}{ vdW } \\
\hline$R_{v}(\AA)$ & 3.7917 & 3.7407 & 3.7727 & 3.7750 \\
\hline$D_{v}(\mathrm{kcal} / \mathrm{mol})$ & 0.0673 & 0.0838 & 0.0661 & 0.0623 \\
\hline$\zeta / \alpha$ & & 12.82 & 16.10 & 16.10 \\
\hline$\zeta_{r}$ & & & & 17.71 \\
\hline $\bar{\kappa}$ & 72.00 & 82.16 & 87.0 & 102.4 \\
\hline \multicolumn{5}{|c|}{ Bond stretch [Eq. (6)] } \\
\hline$R_{b}(\AA)$ & 1.4121 & 1.4165 & 1.4160 & 1.3996 \\
\hline$D_{b}(\mathrm{kcal} / \mathrm{mol})$ & (133.0) & (133.0) & (133.0) & (133.0) \\
\hline$K_{b}\left[(\mathrm{kcal} / \mathrm{mol}) / \AA^{2}\right]$ & 722.12 & 777.15 & 748.80 & 846.59 \\
\hline \multicolumn{5}{|c|}{ Angle bend [Eq. (7)] } \\
\hline$\Theta_{a}(\operatorname{deg})$ & $(120.00)$ & $(120.00)$ & $(120.00)$ & $(120.00)$ \\
\hline$k_{\Theta \Theta}\left[(\mathrm{kcal} / \mathrm{mol}) / \mathrm{rad}^{2}\right]$ & 194.13 & 181.81 & 194.94 & 208.76 \\
\hline$k_{r r}\left[(\mathrm{kcal} / \mathrm{mol}) / \AA^{2}\right]$ & $(68.0)$ & $(68.0)$ & $(68.0)$ & $(68.0)$ \\
\hline$D[(\mathrm{kcal} / \mathrm{mol}) / \AA]$ & 62.67 & 65.61 & 63.87 & 83.22 \\
\hline \multicolumn{5}{|c|}{ Torsion [Eq. (8)] } \\
\hline$V_{t}(\mathrm{kcal} / \mathrm{mol})$ & 22.74 & 21.27 & 21.34 & 22.03 \\
\hline
\end{tabular}

$(-0.50 \%)$, and X6 (+0.38\%) (Table III). A second property sensitive to the vdW parameter is the C33 elastic constant (40.7 $\mathrm{GPa}$ at $0 \mathrm{~K}$ ) which is related to the curvature for the graphene-graphene interactions. Here we find errors of $-4.7 \%$ (LJ12-6), $-0.15 \%$ (Morse), and $+0.25 \%$ (X6), indicating again that the curvature in LJ12-16 potential is too soft. The experimental data on C33 and the $c$ lattice parameter were not used in developing our FF so the excellent correlation validates DHC as a good model for graphite and validates the M06-2X functional for obtaining the correct structure.

The other quite relevant test of the parameters is the interaction energy between graphene planes, $D_{\text {egr-gr }}$. The experimental values for $D_{\text {egr-gr }}$ have varied from 0.18 to $3.92 \mathrm{kcal} / \mathrm{mol}^{44}$ However, a reliable estimate is the recent thermal desorption spectroscopy experiments by Zacharia and co-workers, ${ }^{44}$ where a value of $D_{\text {egr-gr }}=1.19$ $\pm 0.12 \mathrm{kcal} / \mathrm{mol}$ was obtained. Zacharia examined the interactions of various PAH molecules with the basal plane of graphite and estimated the interactions by summing the interactions over the empirical MM2 (Refs. 6 and 7) class FF. This value is in general agreement with work of Benedict and co-workers ${ }^{63}\left(D_{\text {egr-gr }}=0.84 \mathrm{kcal} / \mathrm{mol}\right)$ determined from the interactions of collapsed carbon nanotubes, and a recent quantum Monte Carlo calculation by Spanu et al. ${ }^{64}\left(D_{\text {egr-gr }}\right.$ $=1.3 \pm 0.2 \mathrm{kcal} / \mathrm{mol}$ ). These values are lower than the experimental cohesive energy (at $0 \mathrm{~K}$ ) of polyethylene ${ }^{65}$ of $1.838 \pm 0.032 \mathrm{kcal} / \mathrm{mol}$, and the experimental heat of sublimation (per carbon) of $2.1 \mathrm{kcal} / \mathrm{mol}^{66}$ for benzene and $2.1 \mathrm{kcal} / \mathrm{mol}^{66}$ for $n$-hexane.

Our QMFF-Cx FFs lead to $D_{\text {egr-gr }}=1.22$ (X6), $1.33(\mathrm{LJ})$, and $0.94 \mathrm{kcal} / \mathrm{mol}$ (Morse), in general agreement with the results of Zacharia et al. We included zero point energy corrections at $300 \mathrm{~K}$ by thermodynamics analysis using the optimized cell parameters obtained from the quasiharmonic, free energy minimization (Sec. III E). These zero point corrections were generally small $(<0.02 \mathrm{kcal} / \mathrm{mol}$ at $300 \mathrm{~K})$ and can be neglected. We emphasize again that the agreement with the experimental cohesive energy of graphite is evidence of the accuracy of M06-2X for describing dispersion interactions.

\section{Comparison with other two-body vdW parameters}

Lee and $\mathrm{Kim}^{67}$ developed a potential for polycyclic aromatic hydrocarbons (PAHs) interactions based on the Møller-Plesset second-order perturbation theory ${ }^{56}$ (MP2) using PAHs up to pyrene. This led to $\mathrm{C}-\mathrm{C}$ LJ potential with $R_{e}=3.78 \AA$ and $D_{e}=0.135 \mathrm{kcal} / \mathrm{mol}$, twice the value of our LJ12-6 potential. This is likely because MP2 calculations tend to systematically overbind. ${ }^{54,68}$

Guo et al. ${ }^{69}$ used the experimental C33 elastic constant for the graphene plane interactions plus the $c$ lattice constant at $0 \mathrm{~K}$ to develop a LJ 12-6 description (the GraFF FF), which they used to predict structures of C60 crystal, C70 crystal, and bucky tube systems. Application of the GraFF FF to graphite led to good descriptions of the experimental data. Indeed their 1991 value for $D_{\text {egra-gra }}=1.29 \mathrm{kcal} / \mathrm{mol}$ is 
TABLE III. Calculated properties for graphite at $0 \mathrm{~K}$ (numbers in parenthesis indicate values at $300 \mathrm{~K}$ ). The $A_{2 u}, B_{2 g}$ and $E_{2 g}, E_{1 u}$ experimental lattice modes are used in the FF optimization.

\begin{tabular}{|c|c|c|c|c|c|c|}
\hline & Experiment $(0 \mathrm{~K})^{\mathrm{a}}$ & Fitted? & LJ12-6 & Morse & X6 & $\mathrm{X} 6 \mathrm{~S}$ \\
\hline \multicolumn{7}{|c|}{$\begin{array}{l}\text { Lattice parameters } \\
(\AA)\end{array}$} \\
\hline$a$ & 2.4684 & & 2.4627 & 2.4662 & 2.4741 & 2.4876 \\
\hline$c$ & 6.6721 & & 6.6560 & 6.6406 & 6.6975 & 7.2562 \\
\hline \multicolumn{7}{|c|}{$\begin{array}{c}\text { Elastic constant } \\
\text { (GPa) }\end{array}$} \\
\hline $\mathrm{C} 11$ & $1126 \pm 22$ & & 1127.5 & 1126.9 & 1127.5 & 1126.9 \\
\hline $\mathrm{C} 12$ & $200 \pm 20$ & & 201.8 & 196.1 & 196.1 & 196.0 \\
\hline $\mathrm{C} 33$ & $40.7 \pm 1.1$ & & 38.8 & 40.6 & 40.8 & 18.8 \\
\hline $\mathrm{C} 44$ & $4.51 \pm 0.5$ or $0.18-0.45$ & & 0.220 & 0.330 & 0.227 & 0.826 \\
\hline $\mathrm{C} 13$ & $39.5 \pm 40$ & & 0.230 & 0.330 & 0.218 & 0.226 \\
\hline C66 & $(440)$ & & 465.8 & 465.4 & 465.9 & 465.5 \\
\hline \multicolumn{7}{|c|}{$\begin{array}{l}\text { Young's moduli } \\
\text { (GPa) }\end{array}$} \\
\hline$E_{1}$ & $1020 \pm 30$ & & 1092.3 & 1092.8 & 1093.3 & 1092.7 \\
\hline$E_{3}$ & $36.5 \pm 1.0$ & & 40.7 & 40.6 & 39.7 & 18.2 \\
\hline \multicolumn{7}{|c|}{ Poisson ratios } \\
\hline$\mu_{21}$ & $(0.16 \pm 0.06)$ & & 0.172 & 0.174 & 0.174 & 0.173 \\
\hline$\mu_{31}$ & $(0.34 \pm 0.08)$ & & 0.0002 & 0.0003 & 0.0001 & 0.0001 \\
\hline$\mu_{13}$ & $(0.012 \pm 0.003)$ & & 0.0004 & 0.0067 & 0.0027 & 0.001 \\
\hline \multicolumn{7}{|c|}{$\begin{array}{l}\text { Compressibility } \\
\qquad\left(\mathrm{GPa}^{-1}\right)\end{array}$} \\
\hline$\beta$ & $0.026^{\mathrm{b}}$ & & 0.026 & 0.0261 & 0.027 & 0.056 \\
\hline \multicolumn{7}{|c|}{$\begin{array}{l}\text { Lattice modes } \\
\qquad\left(\mathrm{cm}^{-1}\right)^{\mathrm{c}}\end{array}$} \\
\hline$E_{2 g}$ & $(45)$ & & 11.6 & 12.0 & 11.6 & 0.0 \\
\hline$B_{2 g}$ & (134) & & 139.2 & 136.5 & 136.4 & 85.2 \\
\hline$A_{2 u}$ & 868 & Yes & 869.7 & 868.0 & 867.2 & 868.0 \\
\hline$B_{2 g}$ & 868 & Yes & 870.4 & 868.5 & 868.3 & 868.2 \\
\hline$E_{2 g}$ & $\left(1588^{\mathrm{d}}\right)$ & Yes & 1584.0 & 1582.8 & 1582.2 & 1584.2 \\
\hline$E_{1 u}$ & $\left(1590^{\mathrm{e}}\right)$ & Yes & 1584.2 & 1587.1 & 1588.4 & 1584.3 \\
\hline \multicolumn{7}{|c|}{$\begin{array}{l}\text { Bulk energy } \\
(\mathrm{kcal} / \mathrm{mol} \mathrm{C})^{\mathrm{f}}\end{array}$} \\
\hline$E_{\mathrm{coh}}$ & $1.19 \pm 0.12^{\mathrm{g}}$ & & 1. 33 & 0.94 & 1.22 & 0.80 \\
\hline$E_{\text {hex }}-E_{\text {rhom }}$ & & & 0.003 & 0.001 & 0.002 & 0.0001 \\
\hline \multicolumn{7}{|c|}{$\begin{array}{l}\text { Surface energy } \\
\qquad\left(\mathrm{J} / \mathrm{m}^{2}\right)\end{array}$} \\
\hline$E_{\text {surface }}$ & & & 0.68 & 0.56 & 0.76 & 0.48 \\
\hline
\end{tabular}

${ }^{\mathrm{a}}$ Parentheses indicate values at room temperature.

${ }^{\mathrm{b}}$ Reference 17.

${ }^{\mathrm{c}}$ See discussion in Sec. II C. $8 \mathrm{~cm}^{-1}$ was added to correct from 300 to $0 \mathrm{~K}$.

${ }^{\mathrm{d}}$ Reference 40.

${ }^{\mathrm{e}}$ Reference 39.

${ }^{\mathrm{f}}$ Reference 44.

${ }^{\mathrm{g}}$ These calculations use accuracy bound convergence accuracy parameter of $0.0001 \mathrm{kcal} / \mathrm{mol}$ (Ref. 37), leading to $\eta=1.55 \AA, R_{\text {cut }}=5.55 \AA$, and $H_{\text {cut }}=5.72 \AA^{-1}$ for the hexagonal form with.

within the current experimental value.

The GraFF FF predicts a C44 of $0.401 \mathrm{GPa}$, less than the accepted experimental value of $4.5 \mathrm{GPa}$ from neutron diffraction experiments. There have been several attempts to reproduce this value, primarily using empirical force constants $^{70}$ that couple atoms in adjacent graphene planes.
There is little physical rationalization for such ad hoc terms and no prescription for how to include them when describing graphite interacting with other molecular systems, such as fullerenes, bucky tubes, ${ }^{69}$ solvents, ${ }^{71}$ biological molecules, ${ }^{72}$ and polymers. ${ }^{73}$ Moreover, such interlayer sliding force constants would not provide a way for predicting forces for any 
TABLE IV. Comparison of QMFF-Cx X6 with published vdW parameters for carbon. In each, the valence parameters are adjusted to match the correct experimental in-plane (a) lattice parameter. Errors relative to the experimental value are indicated in brackets for properties sensitive to the vdW.

\begin{tabular}{|c|c|c|c|c|c|c|c|c|}
\hline & QMFF-CX (X6) & $\mathrm{GraFF}^{\mathrm{a}}$ & Dreiding $^{\mathrm{b}}$ & $\mathrm{MM} 2 / \mathrm{MM}^{\mathrm{c}}$ & CHARMM $^{\mathrm{d}}$ & $\mathrm{AMBER}^{\mathrm{e}}$ & Girifalco $^{\mathrm{f}}$ & Ulbricht $^{\mathrm{g}}$ \\
\hline \multicolumn{9}{|c|}{$\begin{array}{c}c \text { lattice parameter } \\
(\AA)\end{array}$} \\
\hline & $\begin{array}{c}6.6975 \\
(+0.38 \%)\end{array}$ & $\begin{array}{c}6.676 \\
(+0.06 \%)\end{array}$ & $\begin{array}{c}6.7773 \\
(+1.51 \%)\end{array}$ & $\begin{array}{c}6.5923 \\
(-1.20 \%)\end{array}$ & $\begin{array}{c}7.009 \\
(+5.05 \%)\end{array}$ & $\begin{array}{c}6.701 \\
(+0.43 \%)\end{array}$ & $\begin{array}{c}6.6732 \\
(+0.02 \%)\end{array}$ & $\begin{array}{c}6.64 \\
(-0.48 \%)\end{array}$ \\
\hline \multicolumn{9}{|c|}{$\begin{array}{l}\text { Elastic constants } \\
\quad(\mathrm{GPa})\end{array}$} \\
\hline C33 & $\begin{array}{c}40.80 \\
(+0.25 \%)\end{array}$ & $\begin{array}{c}40.51 \\
(-0.5 \%)\end{array}$ & $\begin{array}{c}60.13 \\
(+48 \%)\end{array}$ & $\begin{array}{c}24.12 \\
(-41 \%)\end{array}$ & $\begin{array}{l}42.55 \\
(+4 \%)\end{array}$ & $\begin{array}{c}50.24 \\
(+23 \%)\end{array}$ & $\begin{array}{c}49.56 \\
(+21 \%)\end{array}$ & $\begin{array}{c}35.16 \\
(-14 \%)\end{array}$ \\
\hline $\mathrm{C} 44$ & 0.227 & 0.401 & 0.422 & 0.168 & 0.499 & 0.582 & 0.598 & 0.425 \\
\hline $\mathrm{C} 13$ & 0.218 & 0.401 & 0.423 & 0.167 & 0.5 & 0.583 & 0.598 & 0.426 \\
\hline \multicolumn{9}{|c|}{$\begin{array}{l}\text { Cohesive energy } \\
(\mathrm{kcal} / \mathrm{mol} \mathrm{C})\end{array}$} \\
\hline & $\begin{array}{c}1.22 \\
(+2.5 \%)\end{array}$ & $\begin{array}{c}1.3 \\
(+9.2 \%)\end{array}$ & $\begin{array}{c}1.94 \\
(+63 \%)\end{array}$ & $\begin{array}{c}1.021 \\
(-14.2 \%)\end{array}$ & $\begin{array}{c}1.81 \\
(+52.1 \%)\end{array}$ & $\begin{array}{c}2.05 \\
(+72.3 \%)\end{array}$ & $\begin{array}{c}2.04 \\
(+71.4 \%)\end{array}$ & $\begin{array}{c}1.41 \\
(+18.5 \%)\end{array}$ \\
\hline \multicolumn{9}{|c|}{$\begin{array}{c}\text { Compressibility } \beta \\
\left(\mathrm{GPa}^{-1}\right)\end{array}$} \\
\hline & 0.027 & 0.027 & 0.018 & 0.043 & 0.025 & 0.021 & 0.022 & 0.031 \\
\hline \multicolumn{9}{|c|}{$\begin{array}{l}\text { Lattice modes } \\
\qquad\left(\mathrm{cm}^{-1}\right)\end{array}$} \\
\hline$E_{2 g}$ & 11.6 & 13.4 & 13.7 & 13.1 & 14.6 & 16.1 & 16.3 & 13.8 \\
\hline$B_{2 g}$ & 136.4 & 138.7 & 167.2 & 161 & 138.7 & 154.2 & 152.8 & 129.6 \\
\hline$A_{2 u}$ & 867.2 & 867.8 & 1623.3 & & 976 & 1034.6 & 1035.8 & 949.4 \\
\hline \multicolumn{9}{|c|}{ FF parameters vdW } \\
\hline$R_{v}(\AA)$ & 3.7727 & 3.805 & 3.88 & 3.8005 & 3.9848 & 3.816 & 3.8321 & 3.781 \\
\hline$D_{v}(\mathrm{kcal} / \mathrm{mol})$ & 0.0661 & 0.0692 & 0.095 & 0.0515 & 0.07 & 0.086 & 0.0847 & 0.0608 \\
\hline$\zeta$ & 16.1 & $\cdots$ & $\cdots$ & 12.5 & $\cdots$ & $\cdots$ & $\cdots$ & $\cdots$ \\
\hline \multicolumn{9}{|c|}{ Valence } \\
\hline$R_{b}(\AA)$ & 1.416 & 1.4114 & 1.39925 & 1.4219 & 1.38113 & 1.40668 & 1.40577 & 1.40769 \\
\hline
\end{tabular}

${ }^{\mathrm{a}}$ Reference 69 .

${ }^{\mathrm{b}}$ Reference 8 .

${ }^{\mathrm{c}}$ References 6 and 7.

${ }^{\mathrm{d}}$ References 3-5.

${ }^{\mathrm{e}}$ References 1 and 2 .

${ }^{\mathrm{f}}$ Reference 74.

${ }^{\mathrm{g}}$ Reference 75 .

other structure besides the experimental equilibrium structure and hence would not useful in MD/MC (molecular dynamics/Monte Carlo) simulations.

In Table IV we include the properties predicted for graphite from seven other FFs (Refs. 2, 4-6, 8, 74, and 75) and compare them to QMFF-Cx X6 and experiment where applicable. In order to ensure consistent comparisons of the vdW parameters, we use the published vdW parameters and instead readjusted the bond radius $R_{b}$ to obtain zero stress in-plane for the experimental $a$ lattice parameter. Girfalco ${ }^{74}$ and Ulbricht ${ }^{75}$ (the only potentials specifically derived for graphitic systems) did not report any valence interactions, so we assumed harmonic potentials with standard values from the generic Dreiding FF as initial guesses. The optimized potentials for liquid simulations (OPLS) (Ref. 5) nonbond parameters for carbon are similar to those in the CHARMM (Ref. 4) FF so these results are grouped together.
Comparing the calculated $c$ lattice constant of the graphite unit cell, we find the following errors: GraFF (+0.004 ̊), Dreiding (+0.11 ̊), MM2/MM3 (-0.08 $)$, CHARMM/OPLS ( $-0.34 \AA)$, AMBER (+0.03 $\AA)$, Girifalco $(-0.001 \AA)$, and Ulbricht $(+0.03 \AA)$. All parameters except for CHARMM/OPLS could be considered adequate. The errors in the $\mathrm{C} 33$ elastic constant (compared to the experimental value of 40.7$)$ are GraFF $(-0.5 \%)$, Dreiding $(+48 \%)$, MM2/MM3 (-41\%), CHARMM/OPLS (+4\%), AMBER $(+23 \%)$, Girifalco $(+21 \%)$, and Ulbricht $(-14 \%)$. Here, only GraFF leads to errors $<1 \%$, with the CHARMM/OPLS parameters having otherwise acceptable errors. The Dreiding and MM2/MM3 parameters show particularly large errors. Acceptable errors in the experimental cohesive energy $[1.19 \pm 0.2 \mathrm{kcal} / \mathrm{mol}$ (Ref. 44)] are obtained for GraFF $(+9 \%)$, MM2/MM3 $(-14 \%)$, and Ulbricht $(+18 \%)$ poten- 
tials, while Dreiding (+63\%), CHARMM/OPLS $(+52 \%)$, AMBER (+72\%), and Girifalco (+71\%) differ considerably.

Combining all three criteria, the most accurate vdW parameters for carbon from previous FFs is the empirical GraFF FF, with the Ulbricht parameters also acceptable. We consider none of the other previous FFs to be adequate for accurate calculations on graphite.

From our family of QM derived potentials, the X6 potential is the consistently best performer, with errors within the experimental uncertainty for the $c$ lattice constant $(+0.0254 \AA)$, the $\mathrm{C} 33$ elastic constant $(+0.25 \%)$, and the cohesive energy $(+0.25 \%)$. Our Morse potential is also well suited for general simulations and has the benefit of not suffering from the "Buckingham catastrophe," where the energy of the X6 type potentials goes to infinity at short distances. On the other hand, the $1 / R^{6}$ behavior in the X6 should better represent the true physics in this system, as compared to the decaying exponential form of the Morse. We believe that due to the deficiencies pointed out previously, LJ12-6 potentials are limited when applied to graphite and should be avoided.

\section{Graphene-graphene interactions}

We mapped the entire PES for sliding a graphene sheet over another in $0.1 \AA$ increments in the $x$ and $y$ directions (starting from the eclipsed structure). At each displacement, we obtained the interaction energy from the X6 potential, using the large-scale atomic/molecular massively parallel simulator (LAMMPS) (Ref. 76) MD engine. The orthorhombic unit cell was comprised of 96 atoms (48 per sheet), infinitely parallel in the $x y$ plane, with cell dimensions of 5.2 $\times 4.9 \AA^{2}$. The optimal $z$ coordinate at each displacement was obtained by scanning $3.66 \pm 1.0 \AA$, in $0.01 \AA$ increments. The resulting PES and contour map (relative to the PD-X structure) are shown in Fig. 5. X6 predicts a graphite interlayer spacing of $3.333 \AA$ for $\mathrm{ABAB}$ stacking and an increased spacing of $3.413 \AA(+2.4 \%)$ for the eclipsed structure.

We find a rugged PES for sliding of two graphene sheets. Indeed, while the minimum barrier for sliding is $0.0156 \mathrm{kcal} /$ mol C, relative to the PD-X global minima, the sliding motion is not rectilinear. Rather, the minimum energy pathway involves sliding along the edges of the hexagon, from one PD-X-like minimum, through two PD-Y-like local minima, to the next $\mathrm{PD}-\mathrm{X}$. In comparison the barrier is $+0.071 \mathrm{kcal} / \mathrm{mol} \mathrm{C}$ for the $(1,0,0)$ and $(0,1,0)$ directions going over the AA stacked (eclipsed) structure. This eclipsed structure is evident at displacements of $\Delta x= \pm 2.125 \AA$ and $\Delta y= \pm 1.25 \AA$.

\section{Mechanical properties of graphite}

The full set of elastic constants (stiffness $C_{i j}$ and compliance $S_{i j}$ ) are given in Table S.IV (note that only the upper half of the matrices is given).

\section{C44 shear stiffness}

Our X6 calculations lead to $\mathrm{C} 44=0.114$ at $0 \mathrm{~K}$, in good agreement with the mechanical experiments. We conclude that the lower value of $\mathrm{C} 44 \sim 0.18$ is the intrinsic value for

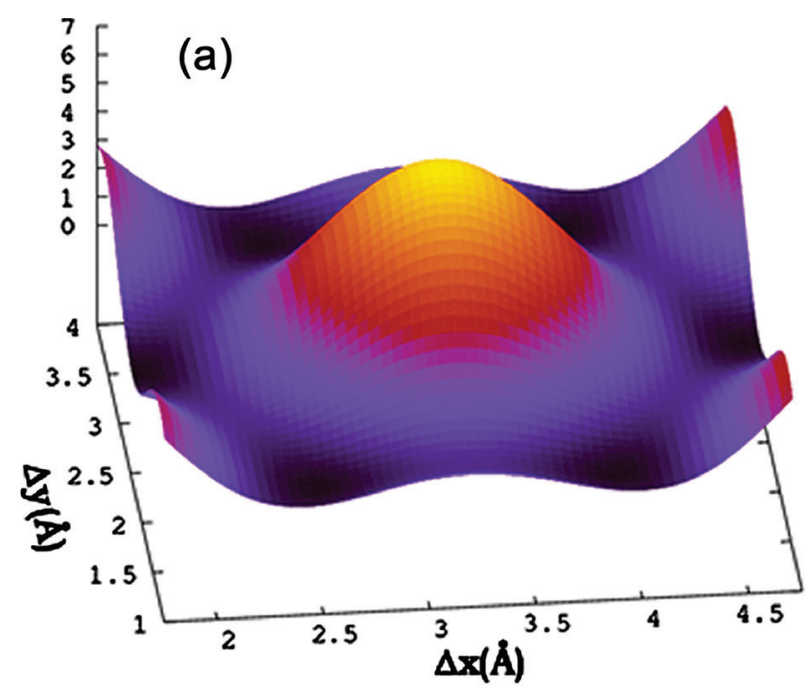

(b)

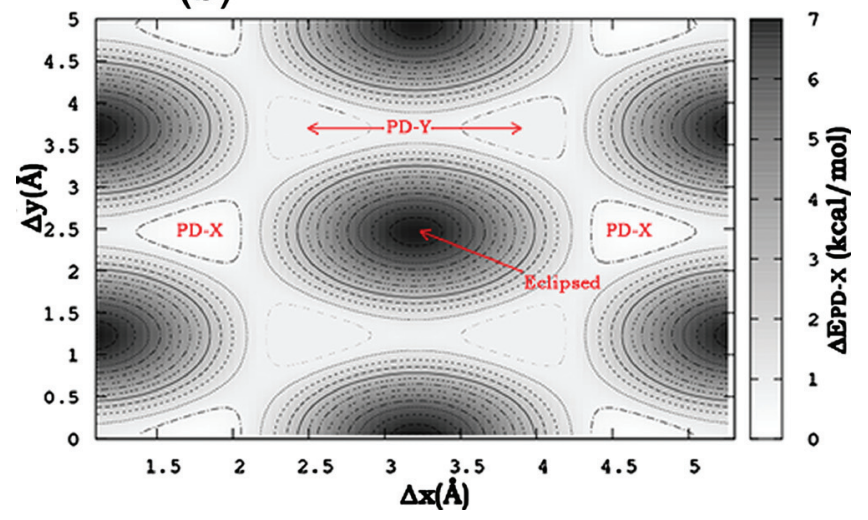

FIG. 5. (a) PES and (b) contour plot for sliding a periodic graphene sheet (96 atoms) over another in $0.1 \AA$ displacements, using the X6 potential. The interplanar distance was optimized at each displacement. All energies are referenced to the PD-X structure, the global minima. The PD-Y structure is a low energy minima $(+0.008 \mathrm{kcal} / \mathrm{mol} \mathrm{C})$. The PD-X $\langle-\rangle \mathrm{PD}-\mathrm{Y}$ barrier is $0.0156 \mathrm{kcal} / \mathrm{mol} \mathrm{C}$ and a low energy pathway for sliding is obtained by tracing the edges of the hexagon unit. The AA stacked eclipsed graphite structure is high energy $(+0.071 \mathrm{kcal} / \mathrm{mol} \mathrm{C})$.

defect free graphite. For highly crystalline graphite the most likely defects are stacking faults between the planes $(\cdots$ ABABABCACAC $\cdots)$ and edge affects due to oxidized exposed edges, rather than dislocations. On the other hand in the inelastic neutron scattering experiments, the neutron irradiation could well lead to defects that couple adjacent graphic planes, increasing the $\mathrm{C} 44$ to the high value observed in the experiments.

\section{C13 stiffness}

We calculate small values for $\mathrm{C} 13(\sim 0.112$ from X6), consistent with flat graphite planes. In all our potentials, the values for $\mathrm{C} 13$ track those of $\mathrm{C} 44$ : Both measures are within $2 \%$. For higher temperatures, the mean fluctuations in crinkling of the sheets should increase, leading to an increased $c$ lattice constant as observed. Applying tension parallel to the sheets might decrease these fluctuations, thereby decreasing the $c$-axis. Thus it is possible that $\mathrm{C} 13$ might be small and constant for low temperature and large for high temperature, as observed. ${ }^{24}$ 


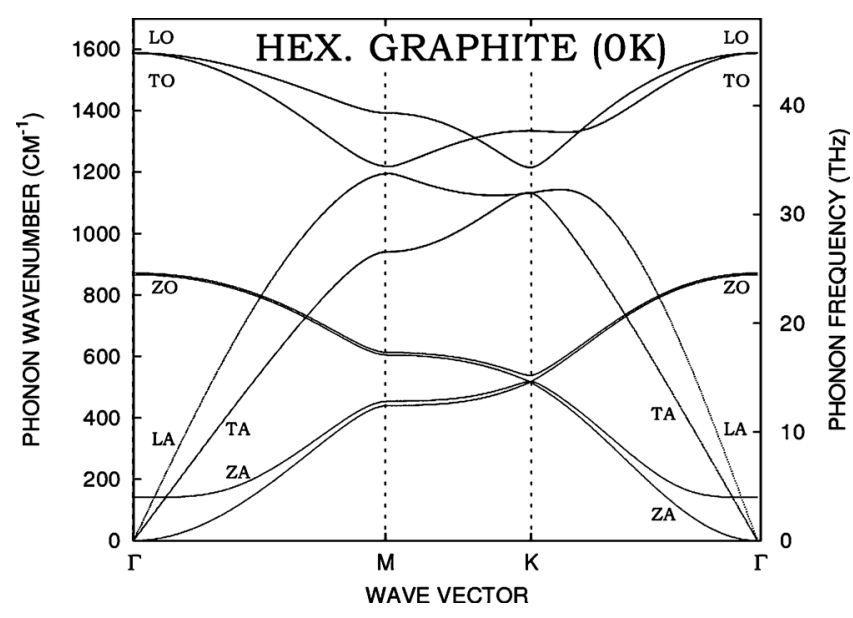

FIG. 6. Phonon dispersion curve for all vibrational modes of (hexagonal) graphite at $0 \mathrm{~K}$ using the $\mathrm{X} 6$ potential.

Consistent with the small calculated value of $\mathrm{C} 13$, we obtain relatively small values for the Poisson ratios coupling strain in the $a$ and $c$ directions at $0 \mathrm{~K}$. While we $\mu_{21}$ $=0.174$ (cf. $0.16 \pm 0.06$ from experiment at $300 \mathrm{~K}$ ), we obtain $\mu_{31}=0.174$, half the experimental value $(0.34 \pm 0.08)$ at $300 \mathrm{~K}$. For stress in the $z$ direction, X6 gives $\mu_{13}=0.0024$, five times less than the $300 \mathrm{~K}$ experimental value of $0.012 \pm 0.003$. Experiments at low temperature on these Poisson ratios would be most valuable in resolving these issues.

\section{Lattice modes}

The CCCC in-plane torsional term in our FF was adjusted to fit the mean value of the out-of-plane crinkling modes (Raman $868 \mathrm{~cm}^{-1}$ and IR $867.7 \mathrm{~cm}^{-1}$ ) in the graphene sheets. This leads to a rotational barrier of $22.74 \mathrm{kcal} / \mathrm{mol}$ for X6 about $1 / 3$ the value for ethylene (65 kcal), as might be expected since there is only one $\pi$ bond for every three $\mathrm{C}-\mathrm{C} \sigma$ bonds. For the two modes at $868 \mathrm{~cm}^{-1}$ we calculate the (Davydov) splitting to be $1.1 \mathrm{~cm}^{-1}$ which is consistent with the experimental value of $<1.0 \mathrm{~cm}^{-1}$. The angle-angle cross terms were adjusted to fit the splitting of the in-plane modes (Raman $1582 \mathrm{~cm}^{-1}$ IR 1587), leading to a Davydov splitting of $\sim 2 \mathrm{~cm}^{-1}$.

The LO mode $\left(B_{2 g}\right)$ is predicted at $141.1 \mathrm{~cm}^{-1}(0 \mathrm{~K})$ for X6 compared to the observed $(300 \mathrm{~K})$ value of $126 \mathrm{~cm}^{-1}$ from neutron scattering. Expansion of the $c$ lattice parameter from the value at $0 \mathrm{~K}$ to the value at $300 \mathrm{~K}$ decreases our predicted value to $v=134 \mathrm{~cm}^{-1}$ at $300 \mathrm{~K}$, explaining most of the discrepancy. The frequency of this mode is directly related to $\mathrm{C} 33$ which we calculate within $0.2 \%$.

The TO mode $\left(E_{2 g}\right)$ is directly related to the C44 elastic constant. Thus neutron-irradiated samples that yield C44 $=4.4$ lead to a much higher frequency $\left(45 \mathrm{~cm}^{-1}\right)$ than the calculations (X6 leads to $\mathrm{TO}=7.4 \mathrm{~cm}^{-1}$ and $\mathrm{C} 44$ $=0.114 \mathrm{GPa}$ ). As discussed above, we believe that the discrepancy may be due to neutron damage. Experimental studies of the TO mode for low temperature and low irradiation would be most valuable to help resolve these uncertainties.

\section{Phonon dispersion curve}

Figure S.1 shows the calculated phonon dispersion curves for the lattice modes of graphite at $0 \mathrm{~K}$. The vibrational frequencies are plotted in terahertz and $\mathrm{cm}^{-1}$ (to convert terahertz to $\mathrm{cm}^{-1}$, multiply by 33.35641 ). The left half is for waves along the $c$-axis, while the right half is for waves along the $a$-axis. The acoustical modes are denoted as TA, ZA, and LA (transverse in-plane, transverse out-ofplane, and longitudinal), while the sheet-sheet optical modes are denoted as TO, ZO, and LO.

The velocity of sound from these calculations for modes along the $c$-axis is

$$
\begin{aligned}
& v_{T}=\sqrt{C_{44} / \rho}=0.44 \mathrm{~km} / \mathrm{s}, \\
& v_{L}=\sqrt{C_{33} / \rho}=4.24 \mathrm{~km} / \mathrm{s},
\end{aligned}
$$

and

$$
\begin{aligned}
& v_{T_{1}}=\sqrt{C_{44} / \rho}=0.44 \mathrm{~km} / \mathrm{s}, \\
& v_{T_{2}}=\sqrt{C_{66} / \rho}=\sqrt{\left(C_{11}-C_{12}\right) / 2 \rho}=13.90 \mathrm{~km} / \mathrm{s}, \\
& v_{L}=\sqrt{C_{11} / \rho}=21.59 \mathrm{~km} / \mathrm{s},
\end{aligned}
$$

for modes along the $a$-axis. Figure 6 shows all the phonon modes. For vibrations within the sheets, there is little dispersion for waves in the $c$ direction.

There are direct experimental data on the lattice modes from neutron scattering; ${ }^{18,19}$ however, these data are for room temperature. To better compare our results with these data, we minimized the cell at $300 \mathrm{~K}$ using the quasiharmonic approximation (Sec. III E). Thus the calculated phonon frequencies take into account anharmonicity due to zero point motions.

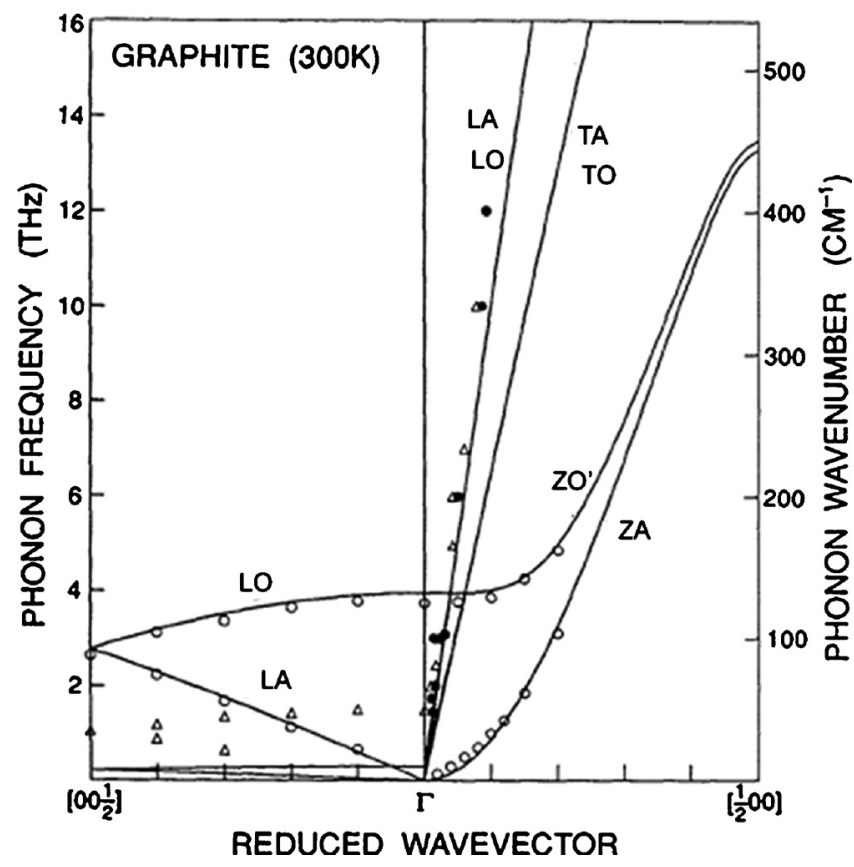

FIG. 7. Phonon dispersion curves for the low frequency modes of (hexagonal) graphite at $300 \mathrm{~K}$. Solid lines from theory and symbols from experimental data (Refs. 18, 19, 42, and 77). 
TABLE V. Phonon frequencies of graphite and derivatives at the high-symmetry points $A, \Gamma, M$, and $K$ in $\mathrm{cm}^{-1}$. The X6 $(0 \mathrm{~K})$ is compared to results from ab initio DFT studies and experiment $(300 \mathrm{~K})$.

\begin{tabular}{|c|c|c|c|c|c|c|}
\hline \multirow{2}{*}{$\begin{array}{l}\text { Functional lattice } \\
\text { constants }\end{array}$} & \multicolumn{3}{|c|}{ Hexagonal } & \multicolumn{2}{|c|}{ Rhombohedral } & \multirow[b]{2}{*}{ Experiment } \\
\hline & X6 & $\mathrm{LDA}^{\mathrm{a}}$ & $\mathrm{GGA}^{\mathrm{a}}$ & X6 & $\mathrm{GGA}^{\mathrm{a}}$ & \\
\hline$a(\AA)$ & 2.4612 & 2.4395 & 2.4607 & 2.4612 & 2.4607 & \\
\hline$c(\AA)$ & 6.7090 & 6.6274 & 6.6274 & 6.7090 & 6.6274 & \\
\hline$A_{\mathrm{TA} / \mathrm{TO}^{\prime}}$ & 10 & 31 & 29 & 12 & & $35^{\mathrm{b}}$ \\
\hline$A_{\mathrm{LA} / \mathrm{LO}^{\prime}}$ & 96 & 80 & 96 & 68 & & $89^{\mathrm{b}}$ \\
\hline$A_{\mathrm{LO}}$ & 825 & 897 & 878 & 864 & & \\
\hline$A_{\mathrm{TO}}$ & 1598 & 1598 & 1564 & 1588 & & \\
\hline$\Gamma_{\mathrm{LO}^{\prime}}$ & 14 & 44 & 41 & 11 & 35 & $49^{\mathrm{b}}$ \\
\hline$\Gamma_{\mathrm{TO}^{\prime}}$ & 138 & 113 & 135 & 118 & 117 & $95^{\mathrm{c}}$ and $126^{\mathrm{a}}$ \\
\hline$\Gamma_{\mathrm{ZO}}$ & 868 & 899 & 879 & 864 & 879 & $861^{\mathrm{c}}$ \\
\hline \multirow[t]{2}{*}{$\Gamma_{\mathrm{LO} / \mathrm{TO}}$} & 1588 & 1593 & 1559 & 1589 & 1559 & $1590^{\mathrm{c}}$ and $1575^{\mathrm{d}}$ \\
\hline & 1589 & 1604 & 1567 & 1604 & & \\
\hline$M_{\mathrm{ZA}}$ & 479 & 478 & 477 & 40 & 479 & $471,{ }^{\mathrm{b}} 465,{ }^{\mathrm{c}}$ and $451^{\mathrm{e}}$ \\
\hline$M_{\mathrm{TA}}$ & 869 & 630 & 626 & 865 & 626 & $630^{\mathrm{e}}$ and $800^{\mathrm{d}}$ \\
\hline$M_{\mathrm{ZO}}$ & 629 & 637 & 634 & 640 & 635 & $670^{\mathrm{c}}$ \\
\hline$M_{\mathrm{LA}}$ & 1341 & 1349 & 1330 & 1388 & 1330 & $1290^{\mathrm{f}}$ \\
\hline$M_{\mathrm{LO}}$ & 1350 & 1368 & 1342 & 1404 & 1344 & $1321^{\mathrm{f}}$ \\
\hline$M_{\mathrm{TO}}$ & 1468 & 1430 & 1394 & 1432 & 1394 & $1388^{\mathrm{f}}$ and $1389^{\mathrm{c}}$ \\
\hline$K_{\mathrm{ZA}}$ & 462 & 540 & 540 & 494 & 535 & $482,{ }^{\mathrm{e}} 517,{ }^{\mathrm{e}}$ and $530^{\mathrm{g}}$ \\
\hline$K_{\mathrm{ZO}}$ & 562 & 544 & 542 & 494 & 539 & $588^{\mathrm{e}}$ and $627^{\mathrm{g}}$ \\
\hline$K_{\mathrm{TA}}$ & 1164 & 1009 & 998 & 1139 & 998 & \\
\hline$K_{\mathrm{LA} / \mathrm{LO}}$ & $1164 / 1280$ & 1239 & 1216 & $1147 / 1157^{\mathrm{h}}$ & 1216 & $1184^{\mathrm{f}}$ and $1202^{\mathrm{f}}$ \\
\hline$K_{\mathrm{TO}}$ & 1424 & 1359 & 1319 & 1292 & 1319 & $1313^{\mathrm{e}}$ and $1291^{\mathrm{g}}$ \\
\hline $\begin{array}{l}{ }^{\mathrm{a}} \text { LDA and GGA D } \\
{ }^{\mathrm{b}} \text { Reference } 19 . \\
{ }^{\mathrm{c}} \text { Reference } 39 . \\
{ }^{\mathrm{d}} \text { Reference } 40 \text { as } \mathrm{r}\end{array}$ & $\begin{array}{l}\text { results frol } \\
\text { orted in Ref }\end{array}$ & $\begin{array}{l}\text { Reference } \\
38 .\end{array}$ & & $\begin{array}{l}\text { eference } 41 \text {. } \\
\text { eference } 42 . \\
\text { eference } 43 \text {. } \\
\text { eference } 39 \text {. }\end{array}$ & & \\
\hline
\end{tabular}

Using this modified X6 potential, we plotted the room temperature lattice modes in Fig. 7 along with the experimental data. For waves in the $a$ direction, there is good agreement with the $\mathrm{ZO}$ and $\mathrm{ZA}$ modes (traverse modes with amplitudes perpendicular to the sheets). The data for longitudinal modes lie slightly higher than the calculated LO and LA modes. This is expected since the experimental dispersion curve from neutron scattering leads to an elastic constant of $\mathrm{C} 11=1440 \mathrm{GPa}$, whereas the experimental value from mechanical studies is $\mathrm{C} 11=1060 \pm 20 \mathrm{GPa}$, in good agreement with X6 C11=1061 GPa.

For waves along the $c$ direction, the predicted LA and LO modes are in good agreement with experiment. As discussed earlier, the TA modes for neutron-irradiated samples lead to a C44 that is 15 times larger than the C44 from mechanical experiments on irradiated samples. Our calculations support the low values for $\mathrm{C} 44$ and lead to TA and TO modes much lower in energy than for neutron experiments.

For the TA mode at the $M k$-point, we find convergence toward $850 \mathrm{~cm}^{-1}$, in good agreement with high-resolution electron energy loss spectroscopy on graphite, ${ }^{39}$ but larger than 626 and $634 \mathrm{~cm}^{-1}$ calculated from LDA and GGA DFT calculations (Table V). The TA and TO modes are apparently not observed in the neutron scattering experiments. Additionally, consistent with a recent inelastic x-ray scattering study by Maultzsch and co-workers, ${ }^{42}$ we find crossing of the LO and TO branches between the $\Gamma-M$ and $\Gamma-K$ directions.

\section{E. Thermodynamic properties of graphite}

The predicted values of various properties using each of these three FFs are listed in Table III for $T>20 \mathrm{~K}$.

\section{Surface energy}

Based on the predicted graphene-graphene interaction energy of $D_{\text {egra-gra }}=1.10 \mathrm{kcal} / \mathrm{mol}$, we estimate that the energy to create two free basal plane surfaces (to form a slab) is $1.52 \mathrm{kcal} / \mathrm{mol}$. To test for additional relaxation effects in forming a fee surface, we calculated the energy for a slab of eight layers, leading to an energy cost of $1.55 \mathrm{kcal} / \mathrm{mol}$. Given the experimental cohesive energy of $1.19 \mathrm{kcal} / \mathrm{mol} \mathrm{C}$, the smallest energy to create a new surface at $0 \mathrm{~K}$ would be $0.625 /\left(\sqrt{3} a^{2} / 4\right)=0.24(\mathrm{kcal} / \mathrm{mol}) / \AA^{2}=1.00(\mathrm{~kJ} / \mathrm{mol}) / \AA^{2}$ $=0.172 \mathrm{~J} / \mathrm{m}^{2}=172 \mathrm{erg} / \mathrm{cm}^{2}$. This is a lower bound on the surface energy. Using the lattice parameters for room temperature leads to an estimated surface energy of $200 \mathrm{erg} / \mathrm{cm}^{2}$ or $0.20 \mathrm{~J} / \mathrm{m}^{2}$. An upper bound on the energy for creating a surface perpendicular to the sheets is $\frac{1}{3}(113) /\left(\frac{1}{2} a c\right)=4.5 \mathrm{kcal} / \mathrm{mol} \AA^{2}=19(\mathrm{~kJ} / \mathrm{mol}) / \AA^{2}$ $=3.2 \mathrm{~J} / \mathrm{m}^{2}=3200 \mathrm{erg} / \mathrm{cm}^{2}$ (assuming each broken bond at the surface costs the average bond energy of $2 / 3$ of the total bulk cohesive energy).

\section{Specific heat capacity}

The entropy $(S)$, vibrational internal energy $(V)$, and free energy $(F)$ are plotted in Fig. S.3. We consider the most 


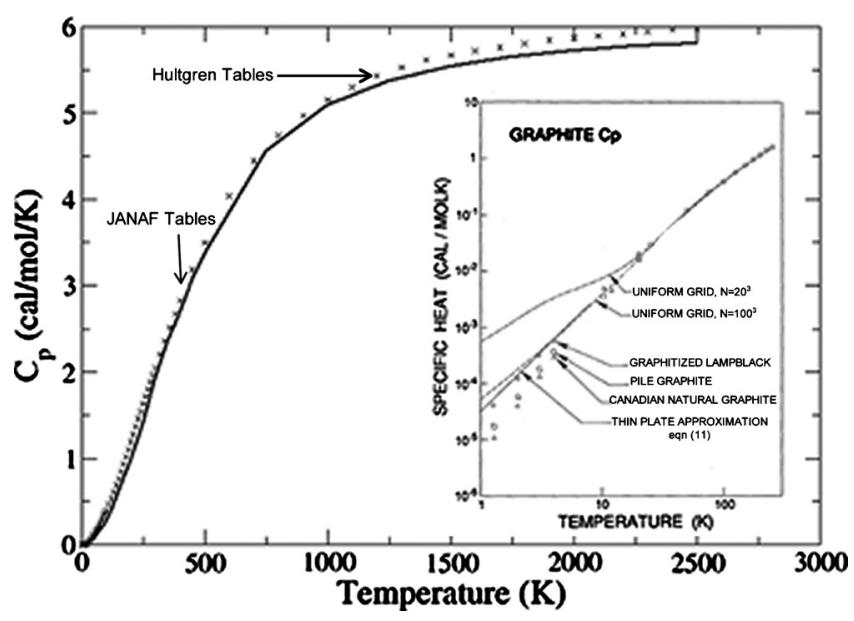

FIG. 8. Specific heat of hexagonal graphite as computed with the QM-FF X6 potential. Low temperature $(<10 \mathrm{~K})$ results obtained from the thin plate approximation, other results obtained from the uniform grid method. Experimental results from different sources are indicated, as reported in Refs. 27 and 46. Values for rhombohedral graphite are not plotted since the lines would be essentially superimposed on the hexagonal graphite lines.

relevant quantity for comparison to experiment is the specific heat capacity. Using a uniform grid, normally $N_{g}=20(8000$ total $k$ points) is sufficient for accurate thermodynamic properties, but for the specific heat we found it necessary to use $N_{g}=100$ (1000 $000 k$ points) to obtain good convergence. We tested whether $N_{g}=100$ was sufficient by using $N_{g}=120$ (1 728000 total points) at $T=10 \mathrm{~K}$. We find that $C_{p}$ increases by $3 \times 10^{-8} \mathrm{cal} / \mathrm{mol} \mathrm{K}$ or $0.08 \%$ (for $N_{g}=20$ it changes by $4.125 \times 10^{-7}$ or $1.1 \%$ ). Based on comparisons to the calculations described below we consider that the $C_{p}$ from $N_{g}=100$ is accurate down to about $2 \mathrm{~K}$.

We also find that the results from the $20 \times 20 \times 20$ grid (dotted lines) differ from the $100 \times 100 \times 100$ grid (solid lines above $T=10 \mathrm{~K}$, dashed lines below) for $T<40 \mathrm{~K}$ (see the inset of Fig. 8). This sensitivity to grid size is primarily due to the low frequency modes in the $z$ direction (see Fig. 6 and Fig. S.1). In particular, neglecting the contribution of the three acoustical modes near the gamma point can decrease $C_{p}$. However the calculated $C_{p}$ for $N_{g}=20$ is too high for $T$ $<10 \mathrm{~K}$. This occurs because only modes with $k_{x}=k_{y}=0$ contribute to $C_{p}$ and the weights of these modes are too large due to the small number of points in the $k_{x}-k_{y}$ plane.

The thin plate results for $T \leq 10 \mathrm{~K}$ are shown in the inset of Fig. 8 by the solid lines. We see that all three experiments and both theoretical cases agree above $20 \mathrm{~K}$. However below $10 \mathrm{~K}$ the experimental results for pile graphite and Canadian natural graphite lead to specific heats significantly below that of graphitized lampblack. It is clear that the calculated optimum parameters for graphite $\mathrm{C} 44=0.11 \mathrm{GPa}$ lead to a $C_{p}$ in excellent agreement with experiment for graphitized lampblack down to $1 \mathrm{~K}$. On the other hand, the calculated $C_{p}$ values are not in agreement with experiment for pile graphite and Canadian natural graphite. These would require a larger $\mathrm{C} 44$, perhaps indicating an increased number of defects.

\section{Lattice parameters and thermal expansion}

For the temperature dependence of the out of plane $c$ lattice parameter, we find an almost linear increase from 0 to
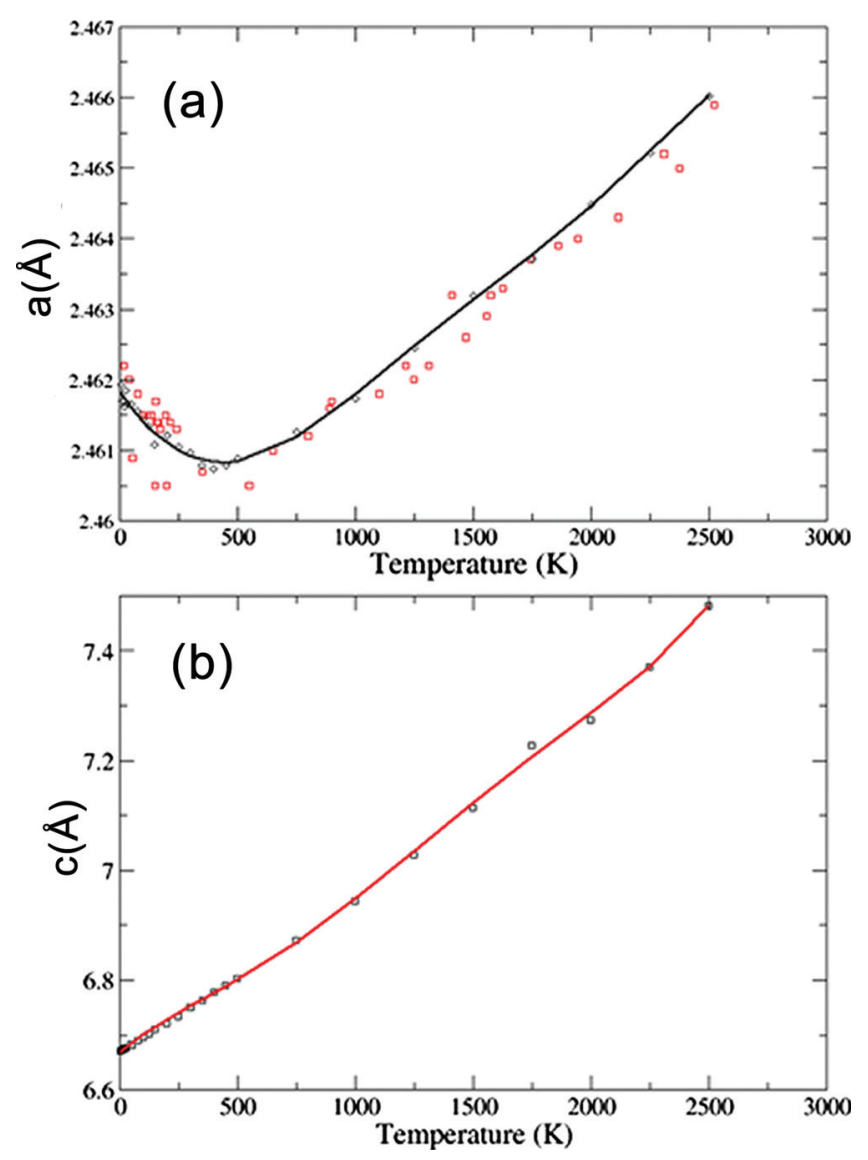

FIG. 9. (a) In-plane (a) lattice parameter of graphite as a function of temperature, calculated with the QM-FF X6 potential. The experimental results (Ref. 52) (red squares) are compared the calculated values (black triangles). The solid black line is the least-squares line to the FF using cubic spline regression. (b) Out of plane $(c)$ lattice parameter of graphite as a function of temperature, calculated with the QM-FF X6 potential. The experimental results (Ref. 52) (red squares) are compared the calculated values (black triangles). The solid black line is the least-squares line by cubic spline regression.

$2500 \mathrm{~K}$ [Fig. 9(b)], in excellent agreement with the experimental results. ${ }^{52}$ The in-plane $a$ lattice parameter is more interesting and provides a good test of the valence forces. Here we find a decrease from 0 to $350 \mathrm{~K}$ with a minimum value of $2.4680 \AA$ at $350 \mathrm{~K}$ [Fig. 9(a)], using the X6 potential. This is consistent to the experimental results of Kellett and Richards ${ }^{52}$ who reported a minimum of $2.4607 \AA$ between 350 and $400 \mathrm{~K}$, and the experimental results of Nelson and Riley ${ }^{78}$ who found $400 \mathrm{~K}$. There was also a theoretical result by Riley ${ }^{79}$ who found $383 \mathrm{~K}$ to be the minimum. From 400 to $2500 \mathrm{~K}$, we observe an almost linear increase in the $a$ lattice parameter. This initial decrease in $a$ is due to the crinkling vibrations of the graphene sheets, which, since the CC bond distances are fixed, shrink the average projected bond distances on the $a b$ plane.

Below room temperature, we find good agreement with the experimental in-plane coefficients of thermal expansion, ${ }^{16,80}$ although the X6 potential predicts a minima at $150 \mathrm{~K}$, whereas the experimental results predict a minima between 200 and $250 \mathrm{~K}$. At the minima, we predict a value of $-1.239 \times 10^{-6} \mathrm{deg}^{-1} \mathrm{~K}$, in excellent agreement with the experimental value of $1.236 \times 10^{-6} \mathrm{deg}^{-1} \mathrm{~K}$. This result is 
not surprising considering the agreement in the specific heat at this temperature observed previously. From 300 to $100 \mathrm{~K}$, the X6 potential overestimates the thermal expansion coefficient by $40 \%$, although convergence is observed at higher temperatures.

\section{F. Rhombohedral graphite}

The planes in graphite stack in the sequence ABABAB $\cdots$, leading to hexagonal symmetry. A second structure with the stacking $\mathrm{ABCABC} \cdots$ and leading to rhombohedral symmetry has also been observed. ${ }^{15}$ From experiment, the hexagonal form is clearly the more stable but an estimate of the difference in energy is not available. Our X6 FF predicts that the rhombohedral form is $0.002 \mathrm{kcal} / \mathrm{mol} \mathrm{C}$ higher than the hexagonal form. The calculated specific heat capacity of rhombohedral graphite is compared with hexagonal graphite in Fig. 8; however the differences are too small to see. Since high quality graphite is hexagonal with but few stacking faults, we believe that the calculated energy difference is reasonable.

The phonon modes for rhombohedral graphite are shown in Fig. S.2. We find a dispersion curve similar to that of hexagonal graphite for frequencies over $400 \mathrm{~cm}^{-1}$. The major discrepancies occur in the $A-\Gamma$ region, with the rhombohedral graphite structure showing an additional LO band in this region.

\section{DISCUSSION}

The experiments and calculations on graphite leave one important discrepancy unresolved: the value of planar sheer elastic constant, C44. Mechanical studies of on bars of highly crystalline graphite lead to $\mathrm{C} 44=0.18-0.33 \mathrm{GPa}$ at room temperature. Our QMFF-Cx X6 FF yields C44 $=0.141(0 \mathrm{~K})$, in good agreement with this experiment. Additionally, the X6 FF predicts a specific heat, $C_{p}$, in agreement with experiments on graphitized lampblack as outlined in Sec. IV E 2.

On the other hand, neutron scattering experiments leads to $\mathrm{C} 44 \sim 4.0 \mathrm{GPa}$. Al-Jishi and Dresselhaus ${ }^{70}$ derived a set of force constants that would lead to $\mathrm{C} 44=4.2 \mathrm{GPa}$ and $\mathrm{C} 33=36.9 \mathrm{GPa}$. However they used a model with valence force constants between atoms in adjacent layers and included individually adjusted radial and tangential pairwise force constants. These pairwise force constants are not derivable from a smooth two-body potential, and could not be used in molecular dynamics simulations.

We propose that the low value of the C44 $(\sim 0.18 \mathrm{GPa})$ is the intrinsic value for defect free graphite. Thus we speculate that neutron-induced defects might couple the planes sufficiently to cause the planes to be "stickier," leading to "pinning" of adjacent planes that might couple the sheets, increasing larger C44 (and C13). Such structures might lead to torsional frequencies smaller than in normal graphite, allowing crinkling that would couple the layers. These defects might also conceivably affect the $868 \mathrm{~cm}^{-1}$ Raman mode.

There are alternative hypotheses for explaining the discrepancy. As the temperature is increased, the graphene planes should exhibit increasing amounts of crinkling, which might increase C44 markedly while affecting C33 only slightly. However, the experimentally observed small change in C44 with temperature ${ }^{24}$ indicates that this is not an important effect.

We should emphasize here that the following assumptions:

(1) that the graphite sheets are flat and

(2) that the vdW interactions are described in terms of twobody functions leading directly to values of C44 $\sim 0.14$ and necessarily leading to

(a) $\mathrm{C} 13 \sim \mathrm{C} 44$,

(b) the high values of $C_{p}$ near $1 \mathrm{~K}$, and

(c) a small energy separation between hexagonal and rhombohedral graphite.

If the high value of $\mathrm{C} 44$ from neutron experiments was the correct value for perfect graphite at $0 \mathrm{~K}$, then either (or both) of the above assumptions (flat sheets or two-body long range attractions) must be false. That is, either the graphite planes are ruffled (as suggested by Pauling) or the vdW interactions cannot described in terms both pairwise additive terms. We do see ruffling of individual graphene planes, ${ }^{81}$ but they are dampened significantly for bulk graphite.

The latter hypothesis is possible. Graphite is a semimetal, i.e., the valence band maximum (highest occupied molecular orbital) is degenerate with the conduction band minimum (lowest unoccupied molecular orbital). In this circumstance, there could be extra large contributions to the dispersion interactions due to the states near the band edges (small energy denominators). This might well have a major effect on C44 (and C13).These effects would not be captured by the M06-2X functional used in this study. They would also increase the vdW energies over that reported here. To describe such effects one would need to include double excitations between occupied and virtual orbitals as in MP2 (Ref. 56) or XYG3 (Ref. 82) methods.

None of our standard three vdW potentials are able to reproduce the M06 energy of the eclipsed structure $(\Delta E=+6.05 \mathrm{kcal} / \mathrm{mol}$ relative to $\mathrm{PD}-\mathrm{X})$, leading to 0.46 for LJ12-6, 1.07 for Morse, and $1.16 \mathrm{kcal} / \mathrm{mol}$ for X6. The eclipsed structure has $\mathrm{CC}$ distances $\sim 0.1 \AA$ smaller than the other structures, suggesting that the FF inner wall is too soft to account for the increase in energy for such a small displacement. To obtain the correct splitting it is necessary to increase the inner wall curvature while keeping the same outer wall parameters as before, which we accomplish using the X6S potential [Eqs. (7)-(9)].

A PD-X/eclipsed splitting of $\Delta E=+6.11 \mathrm{kcal} / \mathrm{mol}$ is obtained for the X6S potential for an inner wall scaling factor of $\chi=1.1$. Reoptimization of the parameters leads to an inner wall parameter of 17.71 , resulting in $\bar{\kappa}=102.4$, or $17 \%$ larger than the original X6. Small changes were observed in the vdW equilibrium distance (increased to 3.775 from 3.773 ) and the binding energy (decreased by $5.8 \%$ from 0.066 ). The valence forces constants also increased to counteract the increased repulsion.

Using this potential, we find significant errors in the 
vdW sensitive parameters of the graphite crystal: a $-54 \%$ error in the $\mathrm{C} 33$ elastic constant and a $+9 \%$ error in the $c$ lattice parameter. The poor performance of this potential in predicting these properties casts doubt on short range Pauli repulsion (exchange) behavior of the M06-2X functional, away from equilibrium.

\section{CONCLUDING REMARKS}

We have obtained a family of simple two-body potentials, based solely on the interactions in the DHC dimer from accurate QM (M06-2X DFT) that describes well the thermodynamic, mechanical, and elastic properties of graphite. The specific heat, thermal expansion, and lattice modes are in good agreement with experiment. We consider that this approach of deriving FF parameters direction from M06-2X QM could be useful for developing new more accurate vdW parameters for other atoms based on the framework.

\section{ACKNOWLEDGMENTS}

This research was partially supported by the Functional Engineered Nano Architects (FENA) via the Microelectronics Advanced Research Corporation (MARCO) with the prime award (2009-NT-2048) at UCLA (PI Kang Wang). T.A.P. thanks the National Science Foundation and the U.S. Department of Energy (CSGF) for graduate fellowships. W.A.G. acknowledges the WCU program at the Korea Advanced Institute of Science and Technology (KAIST) for additional support.

${ }^{1}$ J. M. Wang, R. M. Wolf, J. W. Caldwell, P. A. Kollman, and D. A. Case, J. Comput. Chem. 25, 1157 (2004); D. A. Case, D. A. Pearlman, J. W. Caldwell, T. E. Cheatham, J. Wang, W. S. Ross, C. Simmerling, T. Darden, K. M. Merz, R. V. Stanton, AMBER7, University of California, San Francisco, 1999; A. Perez, I. Marchan, D. Svozil, J. Sponer, T. E. Cheatham, C. A. Laughton, and M. Orozco, Biophys. J. 92, 3817 (2007).

${ }^{2}$ W. D. Cornell, P. Cieplak, C. I. Bayly, I. R. Gould, K. M. Merz, D. M. Ferguson, D. C. Spellmeyer, T. Fox, J. W. Caldwell, and P. A. Kollman, J. Am. Chem. Soc. 117, 5179 (1995).

A. D. MacKerell, D. Bashford, M. Bellott, R. L. Dunbrack, J. D. Evanseck, M. J. Field, S. Fischer, J. Gao, H. Guo, S. Ha, D. Joseph-McCarthy, L. Kuchnir, K. Kuczera, F. T. K. Lau, C. Mattos, S. Michnick, T. Ngo, D. T. Nguyen, B. Prodhom, W. E. Reiher, B. Roux, M. Schlenkrich, J. C. Smith, R. Stote, J. Straub, M. Watanabe, J. Wiorkiewicz-Kuczera, D. Yin, and M. Karplus, J. Phys. Chem. B 102, 3586 (1998); A. D. MacKerell, Jr., J. Wiorkiewicz-Kuczera, and M. Karplus, J. Am. Chem. Soc. 117, 11946 (1995).

${ }^{4}$ B. R. Brooks, R. E. Bruccoleri, B. D. Olafson, D. J. States, S. Swaminathan, and M. Karplus, J. Comput. Chem. 4, 187 (1983).

${ }^{5}$ W. L. Jorgensen and D. L. Severance, J. Am. Chem. Soc. 112, 4768 (1990).

${ }^{6}$ N. L. Allinger, J. Am. Chem. Soc. 99, 8127 (1977).

${ }^{7}$ N. L. Allinger and J. H. Lii, J. Comput. Chem. 8, 1146 (1987).

${ }^{8}$ S. L. Mayo, B. D. Olafson, and W. A. Goddard, J. Phys. Chem. 94, 8897 (1990).

${ }^{9}$ C. D. Sherrill, B. G. Sumpter, M. O. Sinnokrot, M. S. Marshall, E. G. Hohenstein, R. C. Walker, and I. R. Gould, J. Comput. Chem. 30, 2187 (2009).

${ }^{10}$ C. D. Sherrill, T. Takatani, and E. G. Hohenstein, J. Phys. Chem. A 113, 10146 (2009).

${ }^{11}$ Y. Zhao and D. Truhlar, Theor. Chim. Acta 120, 215 (2008).

${ }^{12}$ Y. Zhao, N. E. Schultz, and D. G. Truhlar, J. Chem. Theory Comput. 2, 364 (2006); Y. Zhao and D. G. Truhlar, ibid. 1, 415 (2005); J. Chem. Phys. 125, 194101 (2006).

${ }^{13}$ J. Wu, W. Pisula, and K. Mullen, Chem. Rev. (Washington, D.C.) 107, 718 (2007); S. Stankovich, D. A. Dikin, G. H. B. Dommett, K. M. Kohlhaas, E. J. Zimney, E. A. Stach, R. D. Piner, S. T. Nguyen, and R. S
Ruoff, Nature (London) 442, 282 (2006); L. Venema, ibid. 446, 36 (2007).

${ }^{14}$ Y.-W. Son, M. L. Cohen, and S. G. Louie, Nature (London) 444, 347 (2006); V. Barone, O. Hod, and G. E. Scuseria, Nano Lett. 6, 2748 (2006).

${ }^{15}$ J. Donohue, The Structures of the Elements (Robert E. Krieger, Malabar, FL, 1974)

${ }^{16}$ A. C. Bailey and B. Yates, J. Appl. Phys. 41, 5088 (1970).

${ }^{17}$ O. L. Blakslee, J. Appl. Phys. 41, 3373 (1970).

${ }^{18}$ G. Dolling and B. N. Brockhouse, Phys. Rev. 128, 1120 (1962).

${ }^{19}$ R. Nicklow, H. G. Smith, and N. Wakabaya, Phys. Rev. B 5, 4951 (1972).

${ }^{20}$ C. Baker and A. Kelly, Philos. Mag. 9, 927 (1964).

${ }^{21}$ J. B. Ayasse, C. Ayache, B. Jager, E. Bonjour, and I. L. Spain, Solid State Commun. 29, 659 (1979).

${ }^{22}$ K. Komatsu, J. Phys. Chem. Solids 6, 380 (1958); 25, 707 (1964).

${ }^{23}$ J. C. Bowman and J. A. Krumhansl, J. Phys. Chem. Solids 6, 367 (1958).

${ }^{24}$ W. B. Gauster and I. J. Fritz, J. Appl. Phys. 45, 3309 (1974).

${ }^{25}$ See supplementary material at http://dx.doi.org/10.1063/1.3456543 for the extrapolated $0 \mathrm{~K}$ properties of graphite from experiment, the atomcentered charges of the DHC dimer, the QM DHC energies using 15 different basis sets, the low frequency modes of hexagonal and rhombohedral graphite, calculated at $0 \mathrm{~K}$ and free energies, enthalpies, and entropies as a function of temperature.

${ }^{26}$ R. J. Nemanich, G. Lucovsky, and S. A. Solin, Solid State Commun. 23, 117 (1977).

${ }^{27}$ T. Nihira and T. Iwata, Phys. Rev. B 68, 134305 (2003).

${ }^{28}$ H. O. Pierson, Handbook of Carbon, Graphite, Diamond and Fullerenes: Properties, Processing and Applications (Noyes Publications, Park Ridge, NJ, 1993).

${ }^{29}$ Y. Zhao and D. G. Truhlar, J. Phys. Chem. C 112, 4061 (2008).

${ }^{30}$ D. Feller and K. D. Jordan, J. Phys. Chem. A 104, 9971 (2000).

${ }^{31}$ JAGUAR, version 7.0, Schrödinger, New York, NY, 2007.

${ }^{32}$ W. J. R. Hehre, P. v. R. Schleyer, and J. A. Pople, Ab Initio Molecular Orbital Theory (Wiley, New York, 1986).

${ }^{33}$ S. F. Boys and F. Bernardi, Mol. Phys. 19, 553 (1970); D. W. Schwenke and D. G. Truhlar, J. Chem. Phys. 82, 2418 (1985).

${ }^{34}$ C. P. Kelly, C. J. Cramer, and D. G. Truhlar, Theor. Chem. Acc. 113, 133 (2005).

${ }^{35}$ R. S. Mulliken, J. Chem. Phys. 23, 1833 (1955).

${ }^{36}$ S. Dasgupta and W. A. Goddard, J. Chem. Phys. 90, 7207 (1989).

${ }^{37}$ N. Karasawa and W. A. Goddard, J. Phys. Chem. 93, 7320 (1989).

${ }^{38}$ N. Mounet and N. Marzari, Phys. Rev. B 71, 205214 (2005).

${ }^{39}$ C. Oshima, T. Aizawa, R. Souda, Y. Ishizawa, and Y. Sumiyoshi, Solid State Commun. 65, 1601 (1988).

${ }^{40}$ F. Tuinstra and J. L. Koenig, J. Chem. Phys. 53, 1126 (1970).

${ }^{41}$ H. Yanagisawa, T. Tanaka, Y. Ishida, M. Matsue, E. Rokuta, S. Otani, and C. Oshima, Surf. Interface Anal. 37, 133 (2005).

${ }^{42}$ J. Maultzsch, S. Reich, C. Thomsen, H. Requardt, and P. Ordejon, Phys. Rev. Lett. 92, 075501 (2004).

${ }^{43}$ S. Siebentritt, R. Pues, K. H. Rieder, and A. M. Shikin, Phys. Rev. B 55, 7927 (1997).

${ }^{44}$ R. Zacharia, H. Ulbricht, and T. Hertel, Phys. Rev. B 69, 155406 (2004).

${ }^{45}$ K. Komatsu, J. Phys. Soc. Jpn. 10, 346 (1955).

${ }^{46}$ R. R. Hultgren, Selected Values of the Thermodynamic Properties of the Elements (American Society for Metals, Metals Park, OH, 1973).

${ }^{47}$ W. DeSorbo and W. W. Tyler, J. Chem. Phys. 21, 1660 (1953).

${ }^{48}$ H. Prophet, D. R. Stull, and United States National Bureau of Standards, JANAF Thermochemical Tables (U.S. GPO, Washington, D.C., 1971).

${ }^{49}$ P. Pavone, K. Karch, O. Schutt, W. Windl, D. Strauch, P. Giannozzi, and S. Baroni, Phys. Rev. B 48, 3156 (1993); A. A. Quong and A. Y. Liu, ibid. 56, 7767 (1997); S. Narasimhan and S. de Gironcoli, ibid. 65, 064302 (2002).

${ }^{50}$ N. L. Allan, T. H. K. Barron, and J. A. O. Bruno, J. Chem. Phys. 105, 8300 (1996).

${ }^{51}$ J. D. Gale, J. Chem. Soc., Faraday Trans. 93, 629 (1997); J. D. Gale and A. L. Rohl, Mol. Simul. 29, 291 (2003).

${ }^{52}$ E. A. Kellett and B. P. Richards, J. Nucl. Mater. 12, 184 (1964).

${ }^{53}$ H. Ruuska and T. A. Pakkanen, J. Phys. Chem. B 105, 9541 (2001).

${ }^{54}$ S. Grimme, J. Comput. Chem. 25, 1463 (2004).

${ }^{55}$ S. Grimme, C. Muck-Lichtenfeld, and J. Antony, J. Phys. Chem. C 111, 11199 (2007)

${ }^{56}$ C. Møller and M. S. Plesset, Phys. Rev. 46, 618 (1934).

${ }^{57}$ R. Ditchfield, W. J. Hehre, and J. A. Pople, J. Chem. Phys. 54, 724 
(1971); W. J. Hehre, R. Ditchfie, and J. A. Pople, ibid. 56, 2257 (1972)

${ }^{58}$ T. H. Dunning, J. Chem. Phys. 90, 1007 (1989); R. A. Kendall, T. H. Dunning, and R. J. Harrison, ibid. 96, 6796 (1992).

${ }^{59}$ E. R. Davidson and D. Feller, Chem. Rev. (Washington, D.C.) 86, 681 (1986).

${ }^{60}$ R. E. Easton, D. J. Giesen, A. Welch, C. J. Cramer, and D. G. Truhlar, Theor. Chim. Acta 93, 281 (1996).

${ }^{61}$ J. Friedel, Nuovo Cimento 7, 287 (1958).

${ }^{62}$ T. Dahl, Acta Chem. Scand. 48, 95 (1994).

${ }^{63}$ L. X. Benedict, N. G. Chopra, M. L. Cohen, A. Zettl, S. G. Louie, and V. H. Crespi, Chem. Phys. Lett. 286, 490 (1998).

${ }^{64}$ L. Spanu, S. Sorella, and G. Galli, Phys. Rev. Lett. 103, 196401 (2009).

${ }^{65}$ F. W. Billmeyer, J. Appl. Phys. 28, 1114 (1957).

${ }^{66}$ D. E. Williams and S. R. Cox, Acta Crystallogr., Sect. B: Struct. Sci. 40, 404 (1984).

${ }^{67}$ N. K. Lee and S. K. Kim, J. Chem. Phys. 122, 031102 (2005).

${ }^{68}$ S. Tsuzuki, K. Honda, T. Uchimaru, and M. Mikami, J. Chem. Phys. 120, 647 (2004); Y. Zhao and D. G. Truhlar, J. Chem. Theory Comput. 3, 289 (2007).

${ }^{69}$ Y. J. Quo, N. Karasawa, and W. A. Goddard, Nature (London) 351, 464 (1991)

${ }^{70}$ R. Al-Jishi and G. Dresselhaus, Phys. Rev. B 26, 4514 (1982).
${ }^{71}$ G. Hummer, J. C. Rasaiah, and J. P. Noworyta, Nature (London) 414, 188 (2001).

${ }^{72}$ R. J. Chen, S. Bangsaruntip, K. A. Drouvalakis, N. W. S. Kam, M. Shim, Y. M. Li, W. Kim, P. J. Utz, and H. J. Dai, Proc. Natl. Acad. Sci. U.S.A. 100, 4984 (2003); E. A. Meyer, R. K. Castellano, and F. Diederich, Angew. Chem., Int. Ed. 42, 1210 (2003).

${ }^{73}$ N. Karasawa, S. Dasgupta, and W. A. Goddard, J. Phys. Chem. 95, 2260 (1991).

${ }^{74}$ L. A. Girifalco, M. Hodak, and R. S. Lee, Phys. Rev. B 62, 13104 (2000).

${ }^{75}$ H. Ulbricht, G. Moos, and T. Hertel, Phys. Rev. Lett. 90, 095501 (2003).

${ }^{76}$ S. Plimpton, J. Comput. Phys. 117, 1 (1995).

${ }^{77}$ M. Mohr, J. Maultzsch, E. Dobardzic, S. Reich, I. Milosevic, M. Damnjanovic, A. Bosak, M. Krisch, and C. Thomsen, Phys. Rev. B 76, 035439 (2007).

${ }^{78}$ J. B. Nelson and D. P. Riley, Proc. Phys. Soc. London 57, 477 (1945).

${ }^{79}$ D. P. Riley, Proc. Phys. Soc. London 57, 486 (1945).

${ }^{80}$ W. C. Morgan, Carbon 10, 73 (1972).

${ }^{81}$ A. Fasolino, J. H. Los, and M. I. Katsnelson, Nature Mater. 6, 858 (2007).

${ }^{82}$ Y. Zhang, X. Xu, and W. A. Goddard, Proc. Natl. Acad. Sci. U.S.A. 106, $4963(2009)$ 